\title{
An Analysis of The Effects of Total Quality Management (TQM) on Patient Satisfaction in Hospital: A Scoping Review
}

\author{
Nurul Namirah Kamaruddin ${ }^{1}$, Fridawati Rivai ${ }^{1}$, Andi Zulkifli ${ }^{3}$
}

Email: nurulnamirahks@gmail.com

\author{
${ }^{1}$ Hospital Management Department, Faculty of Public Health, Hasanuddin University, \\ Indonesia \\ ${ }^{2}$ Department of Epidemiology, Faculty of Public Health, Hasanuddin University, Indonesia \\ Received: November 8, $2021 \quad$ Received in Revised: December 15, Accepted: December 26, 2021 \\ 2021
}

\begin{abstract}
Hospitals are an integral part of the health care system. They are accountable for delivering proper health care to very sensitive patients, including all essential difficult medical procedures. Recognizing the wants and aspirations of customers, in this instance patients, is critical for patient happiness. To achieve patient satisfaction, businesses or hospitals must develop and manage a system for acquiring new patients as well as the capacity to keep existing ones. In respect to the quality management system, one instrument that is believed to be capable of assisting organizations in improving their performance and achieving their objectives is Total Quality Management (TQM). As a result, Total Quality Management (TQM) might be one of the strategies adopted. This research technique is referred to as a scoping review. According to the findings of a literature search on the impact of ten TQM components on patient happiness at home, the Focus on Patient factor has the greatest influence on patient satisfaction in hospitals.
\end{abstract}

Keywords: Total Quality Management (TQM), Patient Satisfaction, TQM in Hospital

\section{Introduction}

Nowadays, globalization has reached all aspects of life. Business competition in the current era of globalization is getting tougher, which of course results in rapid changes in a company's environment. The increasingly fierce competition and the increasing number of competitors requires companies to always be able to pay attention to the needs and wants of consumers and try to fulfill them in a way that is superior to what competitors do (Adhiputra, 2019). One of the most effective strategies in global competitiveness is to provide the highest-quality products and services. According to Strong Ismanto, the highest quality is achieved via constant improvement of people skills, processes, and the environment. Businesses may thrive in global competitiveness by focusing on quality. Quality, according to Fandy Tjiptono, is a dynamic state associated with goods, services, people, processes, and the environment that meets expectations (Pardede, 2017).

The government in its function of providing health for the people of Indonesia is based on law number 44 of 2009 concerning hospitals. able to improve services that are more qualified and affordable by the community in order to realize the highest degree of health (UU No.44 Tahun, 2009). Hospitals are a core component of the health sector. They are responsible for providing adequate health care for very sensitive patients with all complex medical procedures when required. The ultimate goal of hospitals, as healthcare providers, is to ensure safe, quality, 
acceptable, efficient and equitable services for their patients (Khawka, 2016). In relation to today's life, health problems have become a basic need for the people of Indonesia. With the increase in people's daily living standards, the demands of the community on the quality of health in hospitals will also increase. This requires health service providers such as hospitals to improve the quality of better services, not only the quality of health services that are curing diseases but also include preventive services to improve the quality of life and provide satisfaction for consumers as users of health services (Masinambow \& Karuntu, 2019).

An organization is said to be of quality service when it is able to provide services in accordance with standards (standard desicion), then can satisfy its customers (customer satisfaction), develops to be said to be of quality if customer delight is not only satisfying, but tends to provide services that are in accordance with standards, providing excellent service. can satisfy its customers, and pay attention to the emotional aspect, namely how the customer's emotional response in receiving the services provided, and always thinking about the innovation aspect by providing services that exceed expectations. Recognizing the objectives and aspirations of customers, in this instance patients, is critical for patient happiness. Satisfied people are a very important asset since they will continue to utilize the services of their choice; on the other hand, unsatisfied consumers will tell others twice as much about their negative experiences.

According to Kotler (2007), patient satisfaction is the degree to which a person feels satisfied with his or her performance (or outcomes) in comparison to his or her expectations. Patient satisfaction, according to Gerson (2004), is the patient's belief that his or her expectations have been fulfilled or surpassed. Overall patient satisfaction is defined as when the patient's personal expectations for treatment and care are fulfilled or surpassed. Vukmir (2006) identifies five characteristics that are strongly associated with patient satisfaction ((Alolayyan et al., 2010), including the following; (1) Waiting before being treated; (2) Nurse's level of concern; (3) Staff organization; (4) Doctor's level of concern; (5) The amount of information provided by the nursing staff. Patient satisfaction will be achieved if the method by which services are delivered from service purchasers to patients is consistent with the customer's perception. This perspective is impacted by subjective elements that might result in discrepancies in perceptions or gaps between consumers and service providers. According to Hafizurrachman (2004), there are five service quality gaps; (1) The gap between management's perception of consumer expectations and service quality specifications; (2) The gap between consumer expectations and management perceptions; (3) The gap between service specifications and the services provided; (4) The gap between actual service delivery and external communication to consumers; (5) The gap between the expected service and the actual service received by consumers.

To achieve patient satisfaction, businesses or hospitals must develop and manage a system for acquiring new patients as well as the capacity to keep existing ones. According to Pohan (2007), service quality has a significant impact on competitiveness in serving health requirements and is critical for the hospital's continued survival. The quality of health services is evaluated not only from a medical technical standpoint, but also from the perspective of the whole health care system, which includes administrative management, finance, equipment, and other health employees (Kafidzin, 2014).

Deming defines quality as the continuous development of a stable system. The definition emphasizes two things, namely all systems must be stable and continuous improvement of various systems to reduce deviations and better meet customer needs. David A. Garfin (1988) in his book Managing Quality: The strategic and competitive edge also suggests eight dimensions of quality, namely performance, features, reliability, conformance, durability, ease 
of use. improvement (Serviceability), aesthetics (Aesthetic), and prepared quality (Perceived Quality). Another approach to quality is "Zero Defect" or without defects proposed by Philip B. Crosby (1979) or making it right the first time (make it right the first time) which is translated into 14 elements of the quality improvement process (Sriwidadi, 2001).

In relation to the quality management system, one tool that is considered to be able to help improve organizational performance to achieve organizational goals is Total Quality Management (TQM). Therefore, Total Quality Management (TQM) can be chosen as one of the methods. In the Health care sector, TQM motivates staff to increase their productivity through high and continuous performance and improvement with staff and patients having full satisfaction through strong strategies and effective plans. TQM is used to improve patient satisfaction, reduce costs and reduce medical errors. The requirements for implementing TQM in organizations Healthcare depends on the organization's managers to make some changes in the structure, processes, work systems, and employees (Alolayyan et al., 2010).

In the abstract level, quality has been defined by 2 important experts in the field of quality, namely Joseph Juran and Edward Deming. According to Juran (1998), Quality is fitness for use, which means that a product or service should be in accordance with what is needed or expected by users. Edward Deming (1986) argues that although quality includes the suitability of product attributes with consumer demands, quality must be more than that (J. Juran \& Godfrey, 1998). Quality Planning is the initial process in a quality management cycle. Juran's (1999) trilogy shows three important interrelated processes, namely quality planning, quality control, and quality improvement. The quality planning stage involves determining customer needs and developing products and processes needed to meet consumer needs. Quality control is an important process to ensure that the realization of production operations is in accordance with the predetermined plan (J. M. Juran et al., 1999)

According to Vincent Gaspersz (2006), Total Quality Management (TQM), quality management (quality management) or integrated quality management (total quality management $=\mathrm{TQM}$ ) is defined as a way of continuously improving management performance at every level of operation, in every functional area. of an organization, using all available human and capital resources. TQM (total quality management) is an extension and development of quality assurance (Quality Assurance). The Quality Assurance Handbook for Higher Education uses the definition of quality assurance as the process of establishing and fulfilling management quality standards in a consistent and sustainable manner, so that consumers, producers and other interested parties obtain satisfaction. Before developing into quality assurance, there was the term Quality control which is one of 3 important interrelated processes according to Juran (Direktorat Penjaminan Mutu, Dirjen Belmawa, 2018). Several quality experts have suggested how to implement TQM based on different approaches. According to Bhat and Cozzalino (1993), there are basically two different approaches. The first is a radical approach taken to correct unnecessary business methods and habits and make the company change drastically. Another approach is incrementally carried out by companies that build quality gradually and gradually. Most of today's TQM implementation is done incrementally because it is essentially a process approach towards quality culture change.

Total Quality Management (TQM) has 10 elements that can be seen as benchmarks or the basis for successful implementation in hospitals. In some previous studies on average only assess one or several elements of TQM. In a study conducted by Reinold and Merlyn (2019), they only looked at the implementation of TQM based on 6 elements, namely customer focus, high obsession, education and training, teamwork, unity of purpose, and involvement and empowerment of health workers (Khawka, 2016). Another study conducted by Zeyad M 
(2016), regarding the detection of TQM status and team work orientation in hospitals, only saw 1 element, namely teamwork which was then linked to its effect on staff satisfaction (Khawka, 2016). Elizabeth M. and Shadrack B. (2019) conducted a study on the application of TQM in service delivery in hospitals with the aim of knowing the effect of customer focus, the influence of empowerment, and the effect of communication on service delivery in hospitals (Mwikali \& Bett, 2019). Babu \& Thomas (2020) also conducted a study that aims to describe the role of leadership in implementing TQM in hospitals and see its relationship with the formation of hospital organizational image (Babu \& Thomas, 2020). Judging from several previous studies, no one has examined the implementation of TQM elements on patient satisfaction, while one indication of improving hospital quality is patient satisfaction. Therefore, researchers want to study further about the implementation of TQM based on 10 elements and see how it affects patient satisfaction in hospitals.

\section{Methods}

This study used a review methodology. This study use a scoping review methodology. The literature search was conducted between June and July 2021, using internet databases (bibliographic searching), specifically in international journals: Science Direct, Google Scholar, and PubMed. Hospital, Patient Satisfaction, and Total Quality Management were utilized as search terms (TQM). The following criteria were used to determine inclusion in this review: (1) Population: Hospitals, patient satisfaction (2) Theoretical Framework: Total Quality Management (3) Context: The influence of Total Quality Management (TQM) implementation on patient satisfaction in hospitals. (4) Review types include quantitative, qualitative, and systematic reviews that were published in English during the previous five years beginning January 1, 2016. (5) If the topics mentioned are not published in journals, the literature will be eliminated. Science Direct, Google Scholar, and PubMed were used to perform initial searches of pertinent online databases. This source was searched and data from January 1, 2016 was obtained. Science Direct, Google Scholar, and PubMed were used to perform initial searches of pertinent online databases. This source was searched and data from January 1, 2016 was obtained.

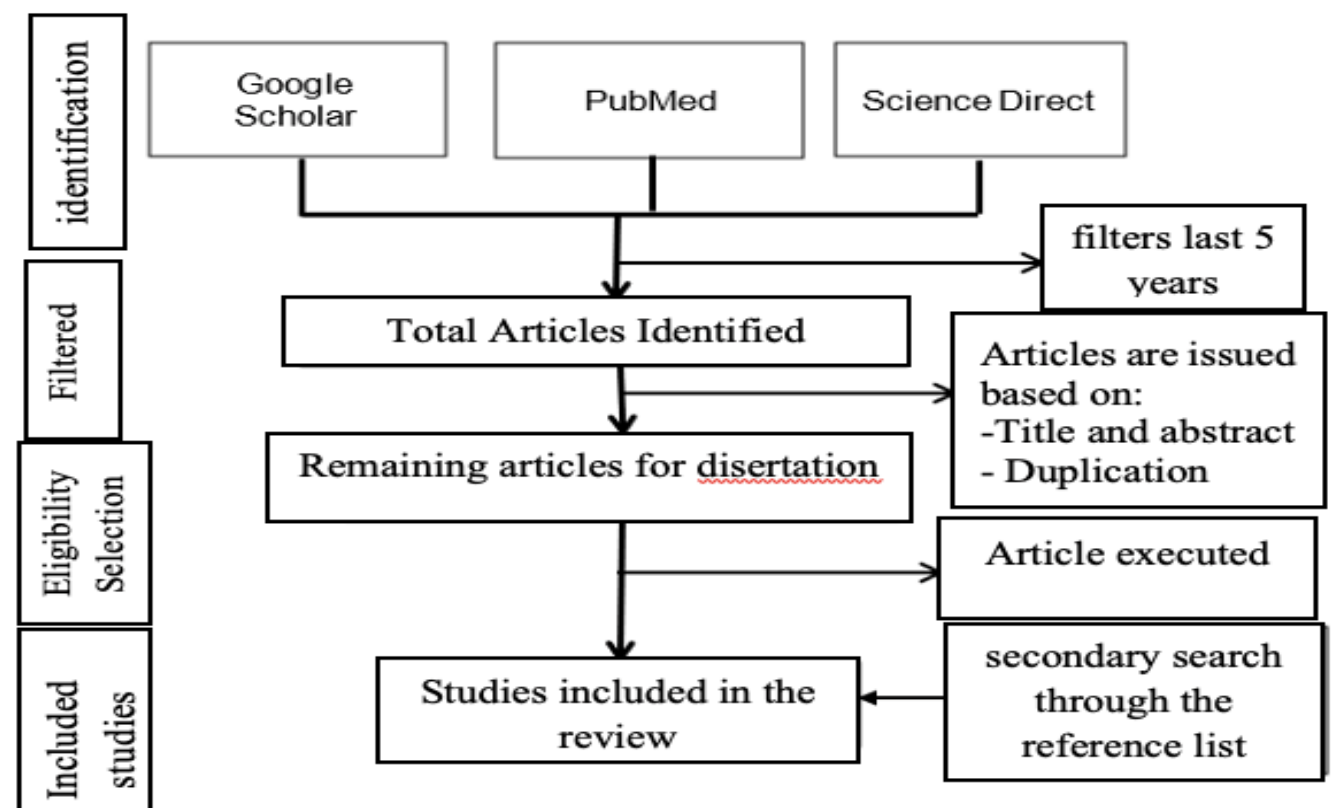

Figure 1. PRISMA Flow Diagram

Copyright $@$ 2021, Journal of Asian Multicultural Research for Medical and Health Science Study, Under the license CC BY-SA 4.0 
Identifying keywords via browsing web databases. patients' satisfaction, population, and hospitals Secondly, the concept of "whole quality management" has been introduced (TQM) The impact of Total Quality Management (TQM) on patient satisfaction in hospitals is examined in this study

\section{Results and Discussion}

A total of 1,206 publications were found in Google Scholar, 805 articles were found in Science Direct, and 855 articles were found in Pubmed, all based on keyword searches. The total number of articles found from the first search was 577 when the results were sorted by publication year beginning with the most recent five years. As a result, the number of published papers was reduced to 46 by a combination of title and abstract selection and improper duplication. According to the inclusion criteria, 25 of the 46 articles were deemed ineligible. Figure 2 depicts the stages involved in data extraction.

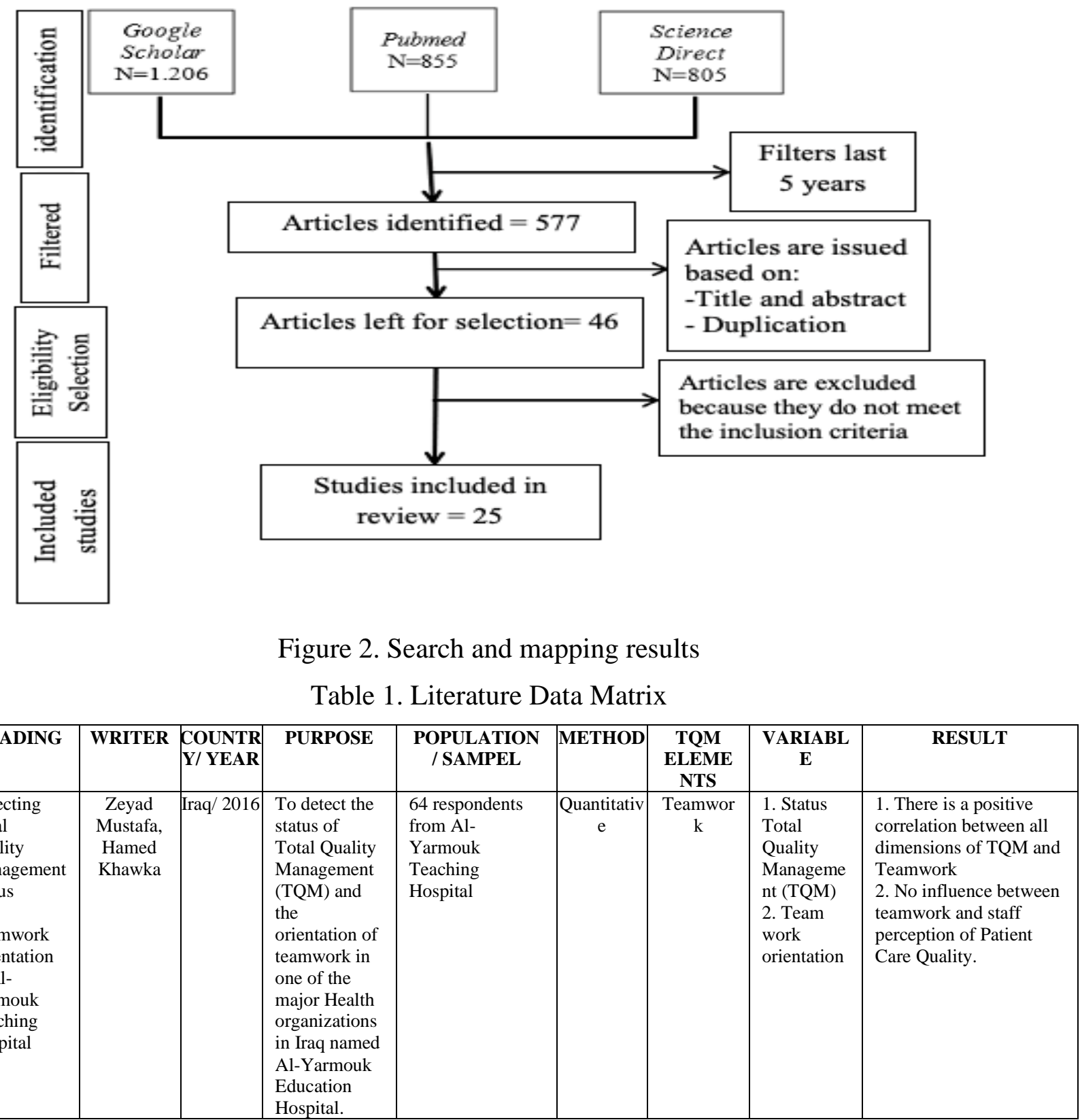




\begin{tabular}{|c|c|c|c|c|c|c|c|c|c|}
\hline 2 & $\begin{array}{l}\text { Total } \\
\text { Quality } \\
\text { Management } \\
\text { Practices } \\
\text { and } \\
\text { Service } \\
\text { Delivery at } \\
\text { The Nairobi } \\
\text { Hospital, } \\
\text { Kenya }\end{array}$ & $\begin{array}{c}\text { Elizabeth } \\
\text { Mwikali, } \\
\text { Shadrack } \\
\text { Bett }\end{array}$ & $\begin{array}{c}\text { Kenya/ } \\
2019\end{array}$ & $\begin{array}{l}\text { To find out } \\
\text { the effect of } \\
\text { TQM } \\
\text { practices on } \\
\text { the delivery } \\
\text { of services at } \\
\text { Nairobi } \\
\text { Hospital }\end{array}$ & $\begin{array}{l}\text { The population } \\
\text { of the study was } \\
1,800 \text { staff of } \\
\text { Nairobi Hospital. } \\
\text { The sample was } \\
180 \text { respondents } \\
\text { from } \\
\text { management } \\
\text { teams, } \\
\text { departments and } \\
\text { operational staff. }\end{array}$ & \begin{tabular}{|c} 
Qualitative \\
$\&$ \\
Quantitativ \\
e
\end{tabular} & $\begin{array}{l}\text { There is } \\
\text { employee } \\
\text { engagem } \\
\text { ent and } \\
\text { empower } \\
\text { ment }\end{array}$ & $\begin{array}{l}\text { 1. TQM } \\
\text { Practice } \\
\text { (hospital } \\
\text { manageme } \\
\text { nt) } \\
\text { 2. Hospital } \\
\text { services }\end{array}$ & $\begin{array}{l}\text { 1. TQM practices } \\
\text { significantly and } \\
\text { positively affect the } \\
\text { delivery of services at } \\
\text { Nairobi Hospital, } \\
\text { Kenya. } \\
\text { 2. Hospitals } \\
\text { significantly embrace } \\
\text { employee empowerment } \\
\text { as a TQM practice by } \\
\text { involving staff in } \\
\text { decision-making and } \\
\text { system improvement. }\end{array}$ \\
\hline 3 & $\begin{array}{l}\text { Total } \\
\text { Quality } \\
\text { Management } \\
\text { Intervention } \\
\text { for } \\
\text { Enhancing } \\
\text { Nursing } \\
\text { Commitmen } \\
\text { t and } \\
\text { Performance } \\
\text { in Jordanian } \\
\text { Hospital: } \\
\text { Protocol of a } \\
\text { Quasi } \\
\text { experimental } \\
\text { study }\end{array}$ & \begin{tabular}{|c|} 
Majdi M. \\
Alzoubi, \\
Hayati K. S., \\
Rosliza A. \\
M., \\
Ahmad A. \\
A., \\
Al-Hamdan \\
Z. M.
\end{tabular} & \begin{tabular}{|c|} 
Yordania/ \\
2018
\end{tabular} & $\begin{array}{l}\text { To develop, } \\
\text { implement, } \\
\text { and evaluate } \\
\text { the influence } \\
\text { of aspects of } \\
\text { Continuous } \\
\text { Quality } \\
\text { Improvement } \\
\text { [CQI] of } \\
\text { TQM } \\
\text { intervention } \\
\text { in improving } \\
\text { the } \\
\text { commitment } \\
\text { and } \\
\text { performance } \\
\text { of nurses at } \\
\text { government } \\
\text { hospitals in } \\
\text { Irbid, Jordan. }\end{array}$ & $\begin{array}{l}\text { The population is } \\
\text { a nurse at a } \\
\text { government } \\
\text { hospital in Irbid, } \\
\text { Jordan } \\
\text { represented by } \\
\text { two hospitals: } \\
\text { Alramtha and } \\
\text { Alraya hospitals. } \\
\text { The total sample } \\
\text { was } 156 \text { nurses } \\
\text { who met the } \\
\text { criteria. }\end{array}$ & Qualitative & $\begin{array}{l}\text { Continuo } \\
\text { us system } \\
\text { improve } \\
\text { ment }\end{array}$ & $\begin{array}{l}1 . \\
\text { Continuous } \\
\text { Quality } \\
\text { Improveme } \\
\text { nt [CQI] } \\
\text { interventio } \\
\text { n TQM } \\
2 . \\
\text { Improved } \\
\text { commitme } \\
\text { nt and } \\
\text { performanc } \\
\text { e of nurses }\end{array}$ & $\begin{array}{l}\text { The commitment and } \\
\text { level of performance of } \\
\text { nurses improved } \\
\text { significantly which } \\
\text { impacted on improving } \\
\text { initiatives and the } \\
\text { quality of health } \\
\text { services.. }\end{array}$ \\
\hline 4 & $\begin{array}{l}\text { Total } \\
\text { Quality } \\
\text { Management } \\
\text { and Hospital } \\
\text { Workforce } \\
\text { National } \\
\text { Cultural } \\
\text { Diversity in } \\
\text { Saudi } \\
\text { Arabia: Help } \\
\text { or } \\
\text { Hindrance? }\end{array}$ & \begin{tabular}{|c|} 
Dr. \\
Abdulrahma \\
n H. \\
Alrabeah, \\
Dr. Susan \\
Ogden, \\
Dr. David \\
Edgar, Dr. \\
Karen Fryer
\end{tabular} & $\begin{array}{c}\text { Saudi } \\
\text { Arabia/ } \\
2020\end{array}$ & $\begin{array}{l}\text { To present } \\
\text { the findings } \\
\text { of an } \\
\text { exploratory } \\
\text { study } \\
\text { investigating } \\
\text { the current } \\
\text { maturity level } \\
\text { of TQM in } \\
\text { Saudi } \\
\text { hospitals and } \\
\text { whether } \\
\text { national } \\
\text { cultural } \\
\text { diversity of } \\
\text { the workforce } \\
\text { (WNCD) is } \\
\text { considered } \\
\text { affect its } \\
\text { development }\end{array}$ & $\begin{array}{l}\text { The population is } \\
\text { the staff of non- } \\
\text { Saudi hospitals in } \\
\text { Saudi Arabia } \\
\text { (Hospitals A, B } \\
\text { and C). } \\
\text { The total sample } \\
\text { was } 939 \text { non- } \\
\text { Saudi staff from } \\
\text { the three } \\
\text { hospitals. }\end{array}$ & $\begin{array}{c}\text { Qualitative } \\
\& \\
\text { Quantitativ } \\
\mathrm{e}\end{array}$ & $\begin{array}{l}\text { 1. Focus } \\
\text { on the } \\
\text { patient } \\
2 . \\
\text { Continuo } \\
\text { us system } \\
\text { improve } \\
\text { ment } \\
3 . \text { Long- } \\
\text { term } \\
\text { commitm } \\
\text { ent } \\
4 . \\
\text { Employe } \\
\mathrm{e} \\
\text { engagem } \\
\text { ent and } \\
\text { empower } \\
\text { ment } \\
5 . \\
\text { Educatio } \\
\mathrm{n} \text { and } \\
\text { training } \\
6 . \\
\text { Obsessio } \\
\mathrm{n} \text { with } \\
\text { quality } \\
7 . \\
\text { Teamwor } \\
\mathrm{k}\end{array}$ & $\begin{array}{l}1 . \\
\text { Determinin } \\
\mathrm{g} \text { factors of } \\
\text { TQM's } \\
\text { success } \\
\text { 2. National } \\
\text { cultural } \\
\text { diversity of } \\
\text { the } \\
\text { workforce }\end{array}$ & $\begin{array}{l}\text { Have a nationally } \\
\text { diverse workforce } \\
\text { generally } \\
\text { It is positive and, by } \\
\text { itself, does not become a } \\
\text { major inhibiting factor } \\
\text { in TQM's success in } \\
\text { hospitals, although there } \\
\text { may be some challenges. }\end{array}$ \\
\hline 5 & $\begin{array}{l}\text { Assessing } \\
\text { Total } \\
\text { Quality } \\
\text { Management } \\
\text { (TQM) } \\
\text { Effect on } \\
\text { Hospital } \\
\text { Performance } \\
\text { in Ghana } \\
\text { Using a } \\
\end{array}$ & $\begin{array}{c}\text { Ronald } \\
\text { Ebenezer } \\
\text { Essel }\end{array}$ & $\begin{array}{c}\text { Ghana/ } \\
2020\end{array}$ & $\begin{array}{l}\text { To explore } \\
\text { the } \\
\text { relationship } \\
\text { between the } \\
\text { implementati } \\
\text { on of TQM's } \\
\text { critical } \\
\text { success } \\
\text { factors and } \\
\text { hospital }\end{array}$ & $\begin{array}{l}\text { The sample was } \\
250 \text { respondents } \\
\text { consisting of } 90 \\
\text { inpatient clients, } \\
100 \text { outpatient } \\
\text { clients, } 60 \\
\text { Hospital staff }\end{array}$ & $\begin{array}{c}\text { Quantitativ } \\
\mathrm{e}\end{array}$ & $\begin{array}{l}\text { 1. Focus } \\
\text { on the } \\
\text { patient } \\
2 . \\
\text { Obsessio } \\
\text { n with } \\
\text { quality } \\
\text { 3. } \\
\text { Scientific } \\
\text { approach }\end{array}$ & $\begin{array}{l}\text { Determinin } \\
\text { g factors of } \\
\text { TQM's } \\
\text { success } \\
\text { 2. Hospital } \\
\text { performanc } \\
\text { e }\end{array}$ & $\begin{array}{l}\text { Significant positive } \\
\text { relationship between all } \\
\text { TQM construction and } \\
\text { hospital performance. } \\
\text { Further findings } \\
\text { revealed that Top } \\
\text { Quality Management } \\
\text { construction contributed } \\
\text { the most to hospital } \\
\text { performance, while }\end{array}$ \\
\hline
\end{tabular}




\begin{tabular}{|c|c|c|c|c|c|c|c|c|c|}
\hline & $\begin{array}{l}\text { Non- } \\
\text { Probabilistic } \\
\text { Approach: } \\
\text { The Case of } \\
\text { Greater } \\
\text { Accra } \\
\text { Regional } \\
\text { Hospital } \\
\text { (GARH) }\end{array}$ & & & $\begin{array}{l}\text { performance } \\
\text { by asking for } \\
\text { the views of } \\
\text { employees } \\
\text { and patients } \\
\text { in Ghana, } \\
\text { with a focus } \\
\text { on Greater } \\
\text { Accra } \\
\text { Regional } \\
\text { Hospital, the } \\
\text { primary } \\
\text { provider of } \\
\text { secondary } \\
\text { public health } \\
\text { services in } \\
\text { Ghana. }\end{array}$ & & & $\begin{array}{l}4 . \\
\text { Educatio } \\
\mathrm{n} \text { and } \\
\text { training } \\
5 . \\
\text { Employe } \\
\mathrm{e} \\
\text { engagem } \\
\text { ent and } \\
\text { empower } \\
\text { ment } \\
6 . \text { Top } \\
\text { Quality } \\
\text { Managem } \\
\text { ent }\end{array}$ & & $\begin{array}{l}\text { training contributed the } \\
\text { least. }\end{array}$ \\
\hline 6 & $\begin{array}{l}\text { The Effect } \\
\text { of 5S- } \\
\text { Continuous } \\
\text { Quality } \\
\text { Improvemen } \\
\text { t-Total } \\
\text { Quality } \\
\text { Management } \\
\text { Approach on } \\
\text { Staff } \\
\text { Motivation, } \\
\text { Patients' } \\
\text { Waiting } \\
\text { Time and } \\
\text { Patient } \\
\text { Satisfaction } \\
\text { with } \\
\text { Services at } \\
\text { Hospitals in } \\
\text { Uganda }\end{array}$ & \begin{tabular}{|} 
Naoki Take, \\
Sarah \\
Byakika, \\
Hiroshi \\
Tasei, Toru \\
Yoshikawa
\end{tabular} & $\begin{array}{l}\text { Uganda/ } \\
2016\end{array}$ & $\begin{array}{l}\text { Analyzed the } \\
\text { effect of } 5 \mathrm{~S} \\
\text { practice on } \\
\text { staff } \\
\text { motivation, } \\
\text { patient } \\
\text { waiting times, } \\
\text { and patient } \\
\text { satisfaction } \\
\text { with } \\
\text { healthcare in } \\
\text { hospitals in } \\
\text { Uganda. }\end{array}$ & $\begin{array}{l}\text { A total of } 4028 \\
\text { and } 2325 \\
\text { samples were } \\
\text { produced from } \\
\text { RRH and GH in } \\
2012-2014 \text { for } \\
\text { patient } \\
\text { satisfaction. For } \\
\text { waiting times, } \\
3,367 \text { and } 3,263 \\
\text { patients were } \\
\text { measured in OPD } \\
\text { and dispensaries } \\
\text { in RRH, and } \\
2126 \text { and } 2092 \\
\text { were performed } \\
\text { in GH. Regarding } \\
\text { staff motivation, } \\
368 \text { and } 216 \text { were } \\
\text { sampled from } \\
\text { RRH and GH. }\end{array}$ & $\begin{array}{c}\text { Quantitativ } \\
\text { e }\end{array}$ & $\begin{array}{l}1 . \\
\text { Continuo } \\
\text { us system } \\
\text { improve } \\
\text { ment } \\
2 . \\
\text { Employe } \\
\text { e } \\
\text { engagem } \\
\text { ent and } \\
\text { empower } \\
\text { ment } \\
3 \text {. Focus } \\
\text { on the } \\
\text { patient } \\
4 . \\
\text { Obsessio } \\
\text { n with } \\
\text { quality }\end{array}$ & $\begin{array}{l}\text { 1. } \\
\text { Continuous } \\
\text { system } \\
\text { improveme } \\
\text { nt } \\
\text { 2. Staff } \\
\text { motivation } \\
\text { 3. Patient } \\
\text { waiting } \\
\text { time } \\
\text { 4. Patient } \\
\text { satisfaction }\end{array}$ & $\begin{array}{l}\text { 5S practices are } \\
\text { effective in supporting } \\
\text { healthcare quality in } \\
\text { terms of staff } \\
\text { motivation, patient } \\
\text { waiting times, and } \\
\text { patient satisfaction. } \\
\text { They also provide two } \\
\text { insights: i) it takes at } \\
\text { least four years to } \\
\text { improve patient } \\
\text { satisfaction through } 5 \mathrm{~S} \\
\text { practice in Uganda, ii) } \\
\text { the fourth year since the } \\
\text { start of } 5 \mathrm{~S} \text { can be the } \\
\text { threshold for } \\
\text { progressing to CQI to } \\
\text { maintain staff } \\
\text { motivation. }\end{array}$ \\
\hline 7 & $\begin{array}{l}\text { A structural } \\
\text { model of } \\
\text { total quality } \\
\text { management } \\
\text {, work } \\
\text { values, job } \\
\text { satisfaction } \\
\text { and patient- } \\
\text { safety- } \\
\text { culture } \\
\text { attitude } \\
\text { among } \\
\text { nurses }\end{array}$ & \begin{tabular}{|c|} 
Kuei Y. \\
Wang, \\
Chuan C. \\
Chou, \\
Jerry C.-Y. \\
Lai
\end{tabular} & $\begin{array}{c}\text { Taiwan/ } \\
2018\end{array}$ & $\begin{array}{l}\text { Explore the } \\
\text { impact of } \\
\text { TQM on } \\
\text { patient safety } \\
\text { culture } \\
\text { attitudes } \\
\text { among } \\
\text { clinical } \\
\text { nurses, } \\
\text { focusing on } \\
\text { correlations } \\
\text { between } \\
\text { TQM, work } \\
\text { values, } \\
\text { employee } \\
\text { satisfaction, } \\
\text { and patient } \\
\text { safety culture } \\
\text { attitudes. } \\
\end{array}$ & $\begin{array}{l}\text { It used comfort } \\
\text { samples from } 30 \\
\text { inpatient units } \\
\text { and } 12 \text { intensive } \\
\text { care units at } \\
\text { taiwan's five } \\
\text { regional teaching } \\
\text { hospitals with } \\
\text { more than } 500 \\
\text { beds. Seven } \\
\text { hundred } \\
\text { questionnaires } \\
\text { were distributed } \\
\text { (140 to each } \\
\text { hospital) during } \\
\text { June 25-July 5, } \\
\text { 2015. }\end{array}$ & $\begin{array}{c}\text { Quantitativ } \\
\text { e }\end{array}$ & $\begin{array}{l}\text { 1. Focus } \\
\text { on the } \\
\text { patient } \\
2 . \\
\text { Teamwor } \\
\mathrm{k} \\
3 . \\
\text { Employe } \\
\text { e } \\
\text { engagem } \\
\text { ent and } \\
\text { empower } \\
\text { ment }\end{array}$ & $\begin{array}{l}\text { 1. Total } \\
\text { Quality } \\
\text { Manageme } \\
\text { nt (TQM) } \\
\text { 2. Work } \\
\text { value } \\
\text { 3. } \\
\text { Employee } \\
\text { satisfaction } \\
\text { 4. Patient } \\
\text { safety } \\
\text { culture } \\
\text { attitude }\end{array}$ & $\begin{array}{l}\text { TQM creates a favorable } \\
\text { work environment and } \\
\text { enhances the culture of } \\
\text { patient safety. TQM, } \\
\text { work value, and } \\
\text { employee satisfaction } \\
\text { orientation are important } \\
\text { predictors of nurse } \\
\text { attitudes toward patient } \\
\text { safety culture attitudes. }\end{array}$ \\
\hline 8 & $\begin{array}{l}\text { Factors } \\
\text { affecting } \\
\text { patient } \\
\text { satisfaction: } \\
\text { an } \\
\text { exploratory } \\
\text { study for } \\
\text { quality } \\
\text { management } \\
\text { in the } \\
\text { health-care } \\
\text { sector }\end{array}$ & \begin{tabular}{|c|} 
Vishal \\
Kamraa, \\
Harjot \\
Singha dan \\
Kalyan \\
Kumar De
\end{tabular} & $\begin{array}{l}\text { India/ } \\
2016\end{array}$ & $\begin{array}{l}\text { The study } \\
\text { examined } \\
\text { factors that } \\
\text { influenced } \\
\text { patient } \\
\text { satisfaction } \\
\text { and its } \\
\text { relationship } \\
\text { to respondent } \\
\text { demographics } \\
\text { for tertiary- } \\
\text { level health } \\
\text { services. }\end{array}$ & $\begin{array}{l}\text { The population is } \\
\text { hospital patients } \\
\text { suffering from } \\
\text { tertiary-level } \\
\text { health diseases in } \\
\text { northern India. } \\
\text { The sample was } \\
883 \text { respondents. }\end{array}$ & $\begin{array}{c}\text { Quantitativ } \\
\text { e }\end{array}$ & $\begin{array}{l}1 . \\
\text { Obsessio } \\
\mathrm{n} \text { with } \\
\text { quality } \\
2 . \\
\text { Teamwor } \\
\mathrm{k} \\
3 . \\
\text { Educatio } \\
\mathrm{n} \text { and } \\
\text { training } \\
4 . \\
\text { Employe } \\
\mathrm{e} \\
\text { engagem } \\
\text { ent and }\end{array}$ & $\begin{array}{l}\text { 1. Patient } \\
\text { satisfaction } \\
\text { 2. Hospital } \\
\text { services } \\
\text { 3. Quality } \\
\text { manageme } \\
\text { nt }\end{array}$ & $\begin{array}{l}\text { Factors that affect } \\
\text { patient satisfaction with } \\
\text { hospital services are } \\
\text { affordability and } \\
\text { comfort, fulfillment of } \\
\text { clinical requirements, } \\
\text { care of nurses and staff, } \\
\text { general behavior of } \\
\text { doctors, registration and } \\
\text { administrative } \\
\text { procedures, } \\
\text { infrastructure and } \\
\text { facilities, professional } \\
\text { behavior. }\end{array}$ \\
\hline
\end{tabular}




\begin{tabular}{|c|c|c|c|c|c|c|c|c|c|}
\hline & & & & & & & $\begin{array}{l}\text { empower } \\
\text { ment }\end{array}$ & & \\
\hline 9 & $\begin{array}{l}\text { The Effect } \\
\text { Of Total } \\
\text { Quality } \\
\text { Management } \\
\text { Practices on } \\
\text { Customer } \\
\text { Satisfaction } \\
\text { in The } \\
\text { Upper West } \\
\text { Regional } \\
\text { Hospital }\end{array}$ & $\begin{array}{l}\text { Mawuko, } \\
\text { D. W. }\end{array}$ & $\begin{array}{c}\text { Ghana/ } \\
2017\end{array}$ & $\begin{array}{l}\text { Assess the } \\
\text { extent of } \\
\text { Total Quality } \\
\text { Management } \\
\text { practices and } \\
\text { their effect on } \\
\text { internal and } \\
\text { external } \\
\text { customer } \\
\text { satisfaction at } \\
\text { Upper West } \\
\text { Hospital. }\end{array}$ & $\begin{array}{l}\text { A total sample of } \\
145 \text { respondents } \\
\text { consisting of } 5 \\
\text { top management, } \\
76 \text { other } \\
\text { employees and } \\
64 \text { patients was } \\
\text { taken from the } \\
\text { population. }\end{array}$ & $\begin{array}{c}\text { Quantitativ } \\
\mathrm{e}\end{array}$ & $\begin{array}{l}\text { 1. Focus } \\
\text { on the } \\
\text { patient } \\
2 . \\
\text { Obsessio } \\
\text { n with } \\
\text { quality } \\
3 \text {. } \\
\text { Employe } \\
\text { e } \\
\text { engagem } \\
\text { ent and } \\
\text { empower } \\
\text { ment }\end{array}$ & $\begin{array}{l}\text { 1. Practice } \\
\text { TQM } \\
2 . \\
\text { Customer } \\
\text { satisfaction }\end{array}$ & $\begin{array}{l}\text { The study's findings } \\
\text { support that Total } \\
\text { Quality Management } \\
\text { practices at Upper West } \\
\text { Regional Hospitals had } \\
\text { no statistically } \\
\text { significant effect on } \\
\text { internal and external } \\
\text { customer satisfaction. }\end{array}$ \\
\hline 10 & $\begin{array}{l}\text { The Impact } \\
\text { of Total } \\
\text { Quality } \\
\text { Management } \\
\text { and } \\
\text { Perceived } \\
\text { Service } \\
\text { Quality on } \\
\text { Patient } \\
\text { Satisfaction } \\
\text { and } \\
\text { Behavior } \\
\text { Intention } \\
\text { in } \\
\text { Palestinian } \\
\text { Healthcare } \\
\text { Organization } \\
\text { s }\end{array}$ & $\begin{array}{l}\text { Ahmed A. } \\
\text { Zaid, Samer } \\
\text { M. Arqawi, } \\
\text { Radwan M. } \\
\text { Abu Mwais, } \\
\text { Mazen J. Al } \\
\text { Shobaki, } \\
\text { Samy S. } \\
\text { Abu-Naser }\end{array}$ & $\begin{array}{c}\text { Palestina/ } \\
2020\end{array}$ & $\begin{array}{l}\text { The study } \\
\text { aims to } \\
\text { investigate } \\
\text { the } \\
\text { relationship } \\
\text { between Total } \\
\text { Quality } \\
\text { Management } \\
\text { (TQM), } \\
\text { perceived } \\
\text { service } \\
\text { quality } \\
\text { (PSQ), as } \\
\text { well as its } \\
\text { impact on } \\
\text { patient } \\
\text { satisfaction } \\
\text { (PS) and } \\
\text { Behavior } \\
\text { Intention (BI) } \\
\text { among } \\
\text { Palestinian } \\
\text { health } \\
\text { organizations. }\end{array}$ & $\begin{array}{l}\text { The survey } \\
\text { population } \\
\text { consisted of } \\
\text { patients from } 40 \\
\text { selected hospitals } \\
\text { in the West Bank } \\
\text { region of } \\
\text { Palestine. } \\
\text { The sample } \\
\text { consisted of } 320 \\
\text { individuals. }\end{array}$ & $\begin{array}{c}\text { Quantitativ } \\
\mathrm{e}\end{array}$ & $\begin{array}{l}\text { 1. Focus } \\
\text { on the } \\
\text { patient } \\
2 . \\
\text { Obsessio } \\
\text { n with } \\
\text { quality }\end{array}$ & $\begin{array}{l}\text { 1. Total } \\
\text { Quality } \\
\text { Manageme } \\
\text { nt (TQM) } \\
2 . \\
\text { Perceived } \\
\text { quality of } \\
\text { service } \\
\text { (PSQ) } \\
\text { 3. Patient } \\
\text { satisfaction } \\
\text { (PS) } \\
\text { 4. } \\
\text { Behavior } \\
\text { Intention } \\
\text { (BI) }\end{array}$ & $\begin{array}{l}\text { There is a significant } \\
\text { relationship between } \\
\text { TQM, PSQ, PS and BI. } \\
\text { Health care quality } \\
\text { factors are closely } \\
\text { related to patient } \\
\text { satisfaction. }\end{array}$ \\
\hline 11 & $\begin{array}{l}\text { TQM and } \\
\text { lean strategy } \\
\text { deployment } \\
\text { in Italian } \\
\text { hospitals: } \\
\text { Benefits } \\
\text { related to } \\
\text { patient } \\
\text { satisfaction } \\
\text { and } \\
\text { encountered } \\
\text { pitfalls }\end{array}$ & $\begin{array}{c}\text { Andrea } \\
\text { Chiarini, } \\
\text { Claudio } \\
\text { Baccarani }\end{array}$ & $\begin{array}{l}\text { Italia, } \\
2016\end{array}$ & $\begin{array}{l}\text { Analyze } \\
\text { deployment } \\
\text { pathways for } \\
\text { total quality } \\
\text { management } \\
\text { (TQM)-Lean } \\
\text { strategies in } \\
\text { public health } \\
\text { care, possible } \\
\text { benefits that } \\
\text { can be } \\
\text { achieved and } \\
\text { difficulties } \\
\text { faced. }\end{array}$ & $\begin{array}{l}\text { Three case } \\
\text { studies were } \\
\text { taken from three } \\
\text { major Italian } \\
\text { hospitals with } \\
\text { more than } 500 \\
\text { beds. The } \\
\text { hospital is } \\
\text { located in } \\
\text { Tuscany, Italy. } \\
\text { These three } \\
\text { hospitals have } \\
\text { embraced TQM } \\
\text { and Lean, } \\
\text { ranging from } \\
\text { strategic } \\
\text { objectives and } \\
\text { their } \\
\text { implementation. }\end{array}$ & Qualitative & $\begin{array}{l}1 . \\
\text { Obsessio } \\
n \text { with } \\
\text { quality } \\
2 \text {. } \\
\text { Continuo } \\
\text { us system } \\
\text { improve } \\
\text { ment } 3 \text {. } \\
\text { There is } \\
\text { employee } \\
\text { engagem } \\
\text { ent and } \\
\text { empower } \\
\text { ment }\end{array}$ & $\begin{array}{l}\text { 1. Total } \\
\text { Quality } \\
\text { Manageme } \\
\text { nt (TQM) } \\
\text { 2. Patient } \\
\text { satisfaction } \\
\text { (PS) } \\
\text { 3. } \\
\text { Difficulties } \\
\text { faced }\end{array}$ & $\begin{array}{l}\text { Hospitals achieve } \\
\text { benefits related to } \\
\text { patient satisfaction and } \\
\text { improved organizational } \\
\text { performance. Issues } \\
\text { related to organizational } \\
\text { and cultural issues, such } \\
\text { as senior manager } \\
\text { commitment, staff } \\
\text { management, } \\
\text { manufacturing culture, } \\
\text { and tool adaptation, can } \\
\text { affect benefits. }\end{array}$ \\
\hline 12 & $\begin{array}{l}\text { The } \\
\text { influence of } \\
\text { total quality } \\
\text { management } \\
\text { on } \\
\text { customer } \\
\text { satisfaction }\end{array}$ & $\begin{array}{c}\text { Nguyen Thi } \\
\text { Le Ha dan } \\
\text { Keisuke } \\
\text { Nagase }\end{array}$ & $\begin{array}{c}\text { Vietnam/ } \\
2019\end{array}$ & $\begin{array}{l}\text { The purpose } \\
\text { of this study } \\
\text { was to find } \\
\text { out the } \\
\text { influence of } \\
\text { Total Quality } \\
\text { Management } \\
\text { by health } \\
\text { facilities on } \\
\text { the perception } \\
\text { of service } \\
\text { quality and } \\
\text { patient } \\
\text { satisfaction. }\end{array}$ & $\begin{array}{l}\text { The population is } \\
2500 \text { inpatients } \\
\text { in various } \\
\text { medical fields } \\
\text { covering } 39 \\
\text { clinical } \\
\text { departments in } \\
\text { Vietnam } \\
\text { Hospitals. } \\
\text { The sample was } \\
550 \text { randomly } \\
\text { selected patients. }\end{array}$ & $\begin{array}{c}\text { Quantitativ } \\
\mathrm{e}\end{array}$ & $\begin{array}{l}\text { 1. Focus } \\
\text { on the } \\
\text { patient } \\
2 . \\
\text { Obsessio } \\
\text { n with } \\
\text { quality }\end{array}$ & $\begin{array}{l}\text { 1. Total } \\
\text { Quality } \\
\text { Manageme } \\
\text { nt (TQM) } \\
\text { 2. Patient } \\
\text { satisfaction }\end{array}$ & $\begin{array}{l}\text { Total Quality } \\
\text { Management has a } \\
\text { significant effect on the } \\
\text { perception of service } \\
\text { quality and patient } \\
\text { satisfaction; Perception } \\
\text { of quality of service } \\
\text { positively affects patient } \\
\text { satisfaction. }\end{array}$ \\
\hline
\end{tabular}




\begin{tabular}{|c|c|c|c|c|c|c|c|c|c|}
\hline 13 & $\begin{array}{l}\text { Total quality } \\
\text { management } \\
: \text { a mediating } \\
\text { factor in the } \\
\text { relationship } \\
\text { between } \\
\text { customer } \\
\text { expectations } \\
\text { and } \\
\text { satisfaction }\end{array}$ & \begin{tabular}{|c|} 
Nguyen Thi \\
Le Ha dan \\
Keisuke \\
Nagase
\end{tabular} & \begin{tabular}{|c|} 
Vietnam, \\
2020
\end{tabular} & $\begin{array}{l}\text { The study } \\
\text { tested Total } \\
\text { Quality } \\
\text { Management } \\
\text { as a factor } \\
\text { that mediated } \\
\text { the } \\
\text { relationship } \\
\text { between } \\
\text { expectations } \\
\text { and customer } \\
\text { satisfaction. }\end{array}$ & $\begin{array}{l}\text { The target } \\
\text { population } \\
\text { consists of } \\
\text { inpatients at } \\
\text { tertiary level } \\
\text { hospitals in } \\
\text { Hanoi, Vietnam. } \\
\text { The sample size } \\
\text { of the study was } \\
\mathrm{N}=500 \text {. }\end{array}$ & $\begin{array}{c}\text { Quantitativ } \\
\mathrm{e}\end{array}$ & $\begin{array}{l}\text { 1. Focus } \\
\text { on the } \\
\text { patient } \\
2 . \\
\text { Obsessio } \\
\text { n with } \\
\text { quality }\end{array}$ & $\begin{array}{l}\text { 1. Total } \\
\text { Quality } \\
\text { Manageme } \\
\text { nt (TQM) } \\
\text { 2. Patient } \\
\text { satisfaction } \\
\text { 3. Patient } \\
\text { expectation } \\
\text { s }\end{array}$ & $\begin{array}{l}\text { Patient expectations } \\
\text { have a significant effect } \\
\text { on total quality } \\
\text { management and patient } \\
\text { satisfaction, while total } \\
\text { quality management has } \\
\text { a positive effect on } \\
\text { satisfaction. Thus, TQM } \\
\text { plays a mediating role in } \\
\text { patient expectations and } \\
\text { satisfaction. }\end{array}$ \\
\hline 14 & $\begin{array}{l}\text { A structural } \\
\text { model of } \\
\text { total quality } \\
\text { management } \\
\text {, kaizen, } \\
\text { operational } \\
\text { performance } \\
\text { on service } \\
\text { quality and } \\
\text { patient } \\
\text { satisfaction }\end{array}$ & \begin{tabular}{|} 
Indra Abadi, \\
Murdifin \\
Haming, \\
Baharuddin \\
S., Amir \\
Mahmud
\end{tabular} & $\begin{array}{c}\text { Indonesia/ } \\
2018\end{array}$ & $\begin{array}{l}\text { Test the } \\
\text { influence of } \\
\text { the strength } \\
\text { of total } \\
\text { quality } \\
\text { management } \\
\text { (TQM), } \\
\text { Kaizen and } \\
\text { operational } \\
\text { performance } \\
\text { on service } \\
\text { quality and } \\
\text { patient } \\
\text { satisfaction. }\end{array}$ & $\begin{array}{l}\text { The population is } \\
\text { a patient in } \\
\text { Makassar City. } \\
\text { The sample was } \\
398 \text { respondents } \\
\text { selected. }\end{array}$ & $\begin{array}{c}\text { Quantitativ } \\
\mathrm{e}\end{array}$ & $\begin{array}{l}\text { 1. Focus } \\
\text { on the } \\
\text { patient } \\
2 . \\
\text { Obsessio } \\
\text { n with } \\
\text { quality } \\
3 . \\
\text { Continuo } \\
\text { us system } \\
\text { improve } \\
\text { ment } \\
4 . \\
\text { Employe } \\
\text { e } \\
\text { engagem } \\
\text { ent and } \\
\text { empower } \\
\text { ment }\end{array}$ & $\begin{array}{l}\text { 1. Total } \\
\text { Quality } \\
\text { Manageme } \\
\text { nt (TQM) } \\
\text { 2. Kaizen } \\
\text { 3. } \\
\text { Operationa } \\
1 \\
\text { performanc } \\
\text { e } \\
\text { 4. Quality } \\
\text { of service } \\
\text { 5. Patient } \\
\text { satisfaction }\end{array}$ & $\begin{array}{l}\text { TQM, Kaizen and } \\
\text { operational performance } \\
\text { are proven to make a } \\
\text { significant positive } \\
\text { contribution to quality } \\
\text { of service and patient } \\
\text { satisfaction. The role of } \\
\text { service quality proved to } \\
\text { be a mediation variable } \\
\text { in analyzing the effect of } \\
\text { TQM, Kaizen and } \\
\text { operational performance } \\
\text { on patient satisfaction. }\end{array}$ \\
\hline 15 & $\begin{array}{l}\text { A research } \\
\text { on the } \\
\text { inpatient } \\
\text { satisfaction } \\
\text { in sivas state } \\
\text { hospital } \\
\text { within the } \\
\text { context of } \\
\text { total quality } \\
\text { management }\end{array}$ & \begin{tabular}{|c|} 
Hatice Genc \\
Kavas, \\
Mehmet \\
Celal \\
Gultekin, \\
Omer Fazil \\
Emek
\end{tabular} & $\begin{array}{l}\text { Turki/ } \\
2016\end{array}$ & $\begin{array}{l}\text { Measure the } \\
\text { effectiveness } \\
\text { and efficiency } \\
\text { of TQM } \\
\text { processes } \\
\text { against } \\
\text { patient } \\
\text { satisfaction. }\end{array}$ & $\begin{array}{l}\text { Population is an } \\
\text { inpatient who is } \\
\text { discharged from } \\
\text { Sivas State } \\
\text { Hospital. The } \\
\text { sample was } 396 \\
\text { patients selected. }\end{array}$ & $\begin{array}{c}\text { Quantitativ } \\
\mathrm{e}\end{array}$ & $\begin{array}{l}\text { 1. Focus } \\
\text { on the } \\
\text { patient } \\
2 . \\
\text { Continuo } \\
\text { us system } \\
\text { improve } \\
\text { ment }\end{array}$ & $\begin{array}{l}\text { 1. Total } \\
\text { Quality } \\
\text { Manageme } \\
\text { nt (TQM) } \\
\text { 2. Patient } \\
\text { satisfaction }\end{array}$ & $\begin{array}{l}\text { It found that hospitals } \\
\text { can use the TQM app } \\
\text { effectively but have } \\
\text { flaws in some things } \\
\text { from a patient's point of } \\
\text { view. }\end{array}$ \\
\hline 16 & $\begin{array}{l}\text { Measuring } \\
\text { the } \\
\text { Influence of } \\
\text { Service } \\
\text { Quality on } \\
\text { Patient } \\
\text { Satisfaction } \\
\text { in Malaysia }\end{array}$ & \begin{tabular}{|c|} 
Christine \\
Nya-Ling \\
Tan, \\
Adedapo \\
Oluwaseyi \\
Ojo, Jun- \\
Hwa Cheah, \\
T. Ramayah
\end{tabular} & $\begin{array}{c}\text { Malaysia/ } \\
2019\end{array}$ & $\begin{array}{l}\text { The study } \\
\text { aims to model } \\
\text { the impact of } \\
\text { quality of } \\
\text { care (medical } \\
\text { care } \\
\text { procedures, } \\
\text { administrativ } \\
\text { e practices, } \\
\text { hospital } \\
\text { image, trust, } \\
\text { patient safety, } \\
\text { infrastructure, } \\
\text { personnel } \\
\text { quality, and } \\
\text { social } \\
\text { responsibility } \\
\text { ) on patient } \\
\text { satisfaction. }\end{array}$ & $\begin{array}{l}\text { The population } \\
\text { are patients from } \\
\text { public and } \\
\text { private hospitals } \\
\text { in Melaka and } \\
\text { Johor. The } \\
\text { sample was } 194 \\
\text { patients selected. }\end{array}$ & $\begin{array}{c}\text { Quantitativ } \\
\mathrm{e}\end{array}$ & $\begin{array}{l}\text { 1. Focus } \\
\text { on the } \\
\text { patient } \\
2 . \\
\text { Obsessio } \\
\mathrm{n} \text { with } \\
\text { quality } \\
3 . \\
\text { Continuo } \\
\text { us system } \\
\text { improve } \\
\text { ment } \\
4 . \\
\text { Educatio } \\
\mathrm{n} \text { and } \\
\text { training } \\
5 . \\
\text { Employe } \\
\mathrm{e} \\
\text { engagem } \\
\text { ent and } \\
\text { empower } \\
\text { ment }\end{array}$ & $\begin{array}{l}\text { 1. Total } \\
\text { Quality } \\
\text { Manageme } \\
\text { nt (TQM) } \\
\text { 2. Patient } \\
\text { satisfaction }\end{array}$ & $\begin{array}{l}\text { Hospital image, patient } \\
\text { safety, personnel } \\
\text { quality, and social } \\
\text { responsibility are } \\
\text { significant predictors of } \\
\text { patient satisfaction. } \\
\text { Personnel quality is the } \\
\text { most important } \\
\text { predictor. The } \\
\text { implication is that in } \\
\text { order for patients to } \\
\text { remain satisfied and } \\
\text { willing to return for } \\
\text { further treatment, } \\
\text { hospitals must invest } \\
\text { more in training their } \\
\text { personnel. }\end{array}$ \\
\hline 17 & $\begin{array}{l}\text { Total } \\
\text { Quality } \\
\text { Management } \\
{[\text { TQM] in }} \\
\text { the } \\
\text { Healthcare } \\
\text { Industry - } \\
\text { Challenges, } \\
\text { Barriers and }\end{array}$ & \begin{tabular}{|c|} 
M. \\
Balasubrama \\
nian
\end{tabular} & $\begin{array}{l}\text { India/ } \\
2016\end{array}$ & $\begin{array}{l}\text { Provide a } \\
\text { framework } \\
\text { for applying } \\
\text { total quality } \\
\text { management } \\
\text { concepts that } \\
\text { are in } \\
\text { accordance } \\
\text { with local }\end{array}$ & $\begin{array}{l}\text { The population is } \\
\text { patients from } \\
\text { Indian hospitals. }\end{array}$ & Qualitative & $\begin{array}{l}\text { 1. Focus } \\
\text { on the } \\
\text { patient } \\
2 \text {. } \\
\text { Obsessio } \\
\text { n with } \\
\text { quality } \\
3 . \\
\text { Continuo }\end{array}$ & $\begin{array}{l}\text { 1. Total } \\
\text { Quality } \\
\text { Manageme } \\
\text { nt (TQM) } \\
\text { 2. Patient } \\
\text { satisfaction } \\
\text { 3. } \\
\text { Employee }\end{array}$ & $\begin{array}{l}\text { The SERVQUAL model } \\
\text { and its application can } \\
\text { assist health facilities in } \\
\text { achieving employee } \\
\text { satisfaction and patient } \\
\text { satisfaction. }\end{array}$ \\
\hline
\end{tabular}




\begin{tabular}{|c|c|c|c|c|c|c|c|c|c|}
\hline & $\begin{array}{l}\text { Implementat } \\
\text { ion } \\
\text { Developing } \\
\text { a } \\
\text { Framework } \\
\text { for TQM } \\
\text { Implementat } \\
\text { ion in a } \\
\text { Healthcare } \\
\text { Setup } \\
\end{array}$ & & & $\begin{array}{l}\text { Indian } \\
\text { culture. }\end{array}$ & & & $\begin{array}{l}\text { us system } \\
\text { improve } \\
\text { ment } \\
4 . \\
\text { Employe } \\
\text { e } \\
\text { engagem } \\
\text { ent and } \\
\text { empower } \\
\text { ment } \\
\end{array}$ & $\begin{array}{l}\text { Satisfactio } \\
\mathrm{n}\end{array}$ & \\
\hline 18 & $\begin{array}{l}\text { Patients' } \\
\text { perceptions } \\
\text { on factors } \\
\text { determining } \\
\text { of Total } \\
\text { Quality } \\
\text { Management } \\
\text { (TQM) } \\
\text { implementat } \\
\text { ion into } \\
\text { Bangladeshi } \\
\text { Public } \\
\text { Hospitals } \\
\text { using } \\
\text { technology: } \\
\text { an empirical } \\
\text { study }\end{array}$ & $\begin{array}{c}\text { Masudur } \\
\text { Rahman, } \\
\text { Valliappan } \\
\text { Raju }\end{array}$ & $\begin{array}{c}\text { Banglades } \\
\text { h/ } 2020\end{array}$ & $\begin{array}{l}\text { To develop } \\
\text { models that } \\
\text { build } \\
\text { relationships } \\
\text { between } \\
\text { TQM practice } \\
\text { and patient } \\
\text { satisfaction, } \\
\text { particularly in } \\
\text { the context of } \\
\text { Bangladesh's } \\
\text { public health } \\
\text { services, } \\
\text { through } \\
\text { empirical } \\
\text { studies. }\end{array}$ & $\begin{array}{l}\text { The population is } \\
\text { an inpatient who } \\
\text { receives medical } \\
\text { care from } 2 \\
\text { tertiary level } \\
\text { hospitals and } 1 \\
\text { middle level } \\
\text { hospital in } \\
\text { Bangladesh. The } \\
\text { sample was } 384 \\
\text { respondents. }\end{array}$ & $\begin{array}{c}\text { Quantitativ } \\
\text { e }\end{array}$ & $\begin{array}{l}\text { 1. Focus } \\
\text { on the } \\
\text { patient } \\
2 \text {. } \\
\text { Obsessio } \\
\text { n with } \\
\text { quality } \\
\text { 3. Long- } \\
\text { term } \\
\text { commitm } \\
\text { ent }\end{array}$ & $\begin{array}{l}\text { 1. Practice } \\
\text { Total } \\
\text { Quality } \\
\text { Manageme } \\
\text { nt (TQM) } \\
\text { 2. Patient } \\
\text { satisfaction }\end{array}$ & $\begin{array}{l}\text { There is a positive and } \\
\text { significant relationship } \\
\text { between TQM practice } \\
\text { and patient satisfaction. } \\
\text { The study also showed } \\
\text { that Top Management } \\
\text { Commitment (TMC) is } \\
\text { considered the most } \\
\text { dominant TQM activity } \\
\text { associated with patient } \\
\text { satisfaction. }\end{array}$ \\
\hline 19 & $\begin{array}{l}\text { Patient } \\
\text { satisfaction } \\
\text { and loyalty } \\
\text { to the } \\
\text { healthcare } \\
\text { organization }\end{array}$ & \begin{tabular}{|c|} 
Nguyen Thi \\
Le Ha dan \\
Keisuke \\
Nagase
\end{tabular} & \begin{tabular}{|c|} 
Vietnam, \\
2020
\end{tabular} & $\begin{array}{l}\text { Tested the } \\
\text { relationship } \\
\text { between } \\
\text { various } \\
\text { factors in the } \\
\text { integrated } \\
\text { model, } \\
\text { including } \\
\text { patient } \\
\text { expectations } \\
\text { (PE), Total } \\
\text { Quality } \\
\text { Management } \\
\text { (TQM), } \\
\text { perceived } \\
\text { service } \\
\text { quality } \\
\text { (PSQ), } \\
\text { patient } \\
\text { satisfaction } \\
\text { (PS), patient } \\
\text { complaints } \\
\text { (PCs) and } \\
\text { patient } \\
\text { loyalty (PL). }\end{array}$ & $\begin{array}{l}\text { The population is } \\
\text { an inpatient who } \\
\text { was admitted in } \\
\text { April } 2018 \text { at a } \\
\text { tertiary level } \\
\text { hospital in } \\
\text { Vietnam. }\end{array}$ & $\begin{array}{c}\text { Quantitativ } \\
\text { e }\end{array}$ & $\begin{array}{l}\text { 1. Focus } \\
\text { on the } \\
\text { patient } \\
2 . \\
\text { Obsessio } \\
\text { n with } \\
\text { quality }\end{array}$ & $\begin{array}{l}\text { 1. Patient } \\
\text { expectation } \\
\text { s (PE) } \\
\text { 2. Total } \\
\text { Quality } \\
\text { Manageme } \\
\text { nt (TQM) } \\
\text { 3. } \\
\text { Perceived } \\
\text { quality of } \\
\text { service } \\
\text { (PSQ) } \\
\text { 4. Patient } \\
\text { satisfaction } \\
\text { (PS) } \\
\text { 5. Patient } \\
\text { complaints } \\
\text { (PC) } \\
\text { 6. Patient } \\
\text { loyalty } \\
\text { (PL). }\end{array}$ & $\begin{array}{l}\text { TQM and PE have a } \\
\text { positive effect on PSQ; } \\
\text { PE affects TQM; PE, } \\
\text { PSQ and TQM affect } \\
\text { PS; PC and PS affect } \\
\text { PL; And PS affects the } \\
\text { PC. }\end{array}$ \\
\hline 20 & $\begin{array}{l}\text { The Impact } \\
\text { of Total } \\
\text { Quality } \\
\text { Management } \\
\text { on Patient } \\
\text { Satısfaction: } \\
\text { A Field } \\
\text { Study in the } \\
\text { Health } \\
\text { Centers of } \\
\text { the Syrians } \\
\text { in Gazıantep }\end{array}$ & \begin{tabular}{|c|} 
Abdulkadir \\
Bilen, Ahmet \\
Sitki
\end{tabular} & $\begin{array}{c}\text { Suriah/ } \\
2020\end{array}$ & $\begin{array}{l}\text { To find out } \\
\text { patient } \\
\text { satisfaction in } \\
\text { the field of } \\
\text { health } \\
\text { represented } \\
\text { by the TQM } \\
\text { indicator. } \\
\text { Includes the } \\
\text { impact of } \\
\text { total quality } \\
\text { management } \\
\text { factors on } \\
\text { patient } \\
\text { satisfaction. }\end{array}$ & $\begin{array}{l}\text { The population is } \\
\text { Syrians who } \\
\text { receive services } \\
\text { from health } \\
\text { centers. The } \\
\text { sample was } 88 \\
\text { respondents. }\end{array}$ & Qualitative & $\begin{array}{l}\text { 1. Focus } \\
\text { on the } \\
\text { patient } \\
2 . \text { Long- } \\
\text { term } \\
\text { commitm } \\
\text { ent } \\
3 \text {. } \\
\text { Teamwor } \\
\mathrm{k} \\
4 . \\
\text { Continuo } \\
\text { us system } \\
\text { improve } \\
\text { ment } \\
5 . \\
\text { Employe } \\
\text { e } \\
\text { engagem } \\
\text { ent and }\end{array}$ & $\begin{array}{l}\text { 1. Total } \\
\text { Quality } \\
\text { Manageme } \\
\text { nt (TQM) } \\
\text { 2. Patient } \\
\text { satisfaction }\end{array}$ & $\begin{array}{l}\text { Patient satisfaction } \\
\text { increases as total quality } \\
\text { management practices } \\
\text { increase. }\end{array}$ \\
\hline
\end{tabular}




\begin{tabular}{|c|c|c|c|c|c|c|c|c|c|}
\hline & & & & & & & $\begin{array}{l}\text { empower } \\
\text { ment }\end{array}$ & & \\
\hline 21 & $\begin{array}{l}\text { Total } \\
\text { Quality } \\
\text { Management } \\
\text { \& Customer } \\
\text { Satisfaction } \\
\text { in Public } \\
\text { Hospitals in } \\
\text { Sri Lanka }\end{array}$ & \begin{tabular}{|c|} 
H. K. Pemal \\
Daksith, \\
Uthpalee \\
Hewage
\end{tabular} & $\begin{array}{c}\text { Sri Lanka/ } \\
2021\end{array}$ & $\begin{array}{l}\text { To evaluate } \\
\text { the } \\
\text { relationship } \\
\text { between the } \\
\text { principles of } \\
\text { Total Quality } \\
\text { Management } \\
\text { (TQM) and } \\
\text { Customer } \\
\text { Satisfaction } \\
\text { of the } \\
\text { Department } \\
\text { of Outpatients } \\
\text { (OPD) in Sri } \\
\text { Lankan } \\
\text { public } \\
\text { hospitals }\end{array}$ & $\begin{array}{l}\text { The population } \\
\text { are patients and } \\
\text { employees of the } \\
\text { Outpatient } \\
\text { Department at Sri } \\
\text { Lanka's public } \\
\text { hospitals. The } \\
\text { sample was } 207 \\
\text { respondents. }\end{array}$ & Qualitative & $\begin{array}{l}\text { 1. Focus } \\
\text { on the } \\
\text { patient } \\
2 . \text { Long- } \\
\text { term } \\
\text { commitm } \\
\text { ent } \\
3 \text {. } \\
\text { Teamwor } \\
\mathrm{k} \\
4 . \\
\text { Educatio } \\
\mathrm{n} \text { and } \\
\text { training } \\
5 \text {. } \\
\text { Employe } \\
\mathrm{e} \\
\text { engagem } \\
\text { ent and } \\
\text { empower } \\
\text { ment }\end{array}$ & $\begin{array}{l}\text { 1. Total } \\
\text { Quality } \\
\text { Manageme } \\
\text { nt (TQM) } \\
\text { 2. Patient } \\
\text { satisfaction } \\
\text { (CS) }\end{array}$ & $\begin{array}{l}\text { The results of the } \\
\text { analysis showed that } \\
\text { TQM had a significant } \\
\text { positive impact on } \\
\text { patient satisfaction in the } \\
\text { outpatient process in } \\
\text { public hospitals. }\end{array}$ \\
\hline 22 & $\begin{array}{l}\text { The } \\
\text { availability } \\
\text { of Total } \\
\text { Quality } \\
\text { Management } \\
\text { requirements } \\
\text { and } \\
\text { contribution } \\
\text { to the } \\
\text { achievement } \\
\text { of the } \\
\text { quality of } \\
\text { service } \\
\text { health } \\
\text { Analytical } \\
\text { study at } \\
\text { Salah al-Din } \\
\text { General } \\
\text { Hospital }\end{array}$ & \begin{tabular}{|c|} 
Assist. Prof. \\
Kefah Abbas \\
Muhaimeed, \\
Assist. Prof. \\
Dr. Thamer \\
Akkab \\
Hawas, \\
Lecturer Dr. \\
Ammar \\
Awad \\
Mohammed
\end{tabular} & Iraq/ 2021 & $\begin{array}{l}\text { To identify } \\
\text { the extent of } \\
\text { TQM's } \\
\text { availability } \\
\text { and its } \\
\text { contribution } \\
\text { to achieving } \\
\text { quality of } \\
\text { service to } \\
\text { ensure high- } \\
\text { quality } \\
\text { healthcare } \\
\text { that satisfies } \\
\text { patients }\end{array}$ & $\begin{array}{l}\text { The population is } \\
\text { workers at Salah } \\
\text { al-Din General } \\
\text { Hospital in Salah } \\
\text { al-Din Province. } \\
\text { The sample was } \\
30 \text { respondents. }\end{array}$ & Qualitative & $\begin{array}{l}\text { 1. Focus } \\
\text { on the } \\
\text { patient } \\
2 . \\
\text { Obsessio } \\
\text { n with } \\
\text { quality } \\
3 . \\
\text { Employe } \\
\text { e } \\
\text { engagem } \\
\text { ent and } \\
\text { empower } \\
\text { ment }\end{array}$ & $\begin{array}{l}\text { 1. Total } \\
\text { Quality } \\
\text { Manageme } \\
\text { nt (TQM) } \\
\text { 2. Quality } \\
\text { of health } \\
\text { care } \\
\text { 3. Patient } \\
\text { satisfaction }\end{array}$ & $\begin{array}{l}\text { Hospitals should pay } \\
\text { attention to TQM } \\
\text { requirements, as these } \\
\text { requirements are } \\
\text { necessary to achieve } \\
\text { quality of service and } \\
\text { thus achieve patient } \\
\text { satisfaction. }\end{array}$ \\
\hline 23 & $\begin{array}{l}\text { Measuring } \\
\text { the impact } \\
\text { of soft and } \\
\text { hard total } \\
\text { quality } \\
\text { management } \\
\text { factors on } \\
\text { customer } \\
\text { behavior } \\
\text { based on the } \\
\text { role of } \\
\text { innovation } \\
\text { and } \\
\text { continuous } \\
\text { improvemen } \\
\mathrm{t}\end{array}$ & \begin{tabular}{|} 
Mohammad \\
Javad \\
Ershadi , \\
Nafiseh \\
Najafi, Paria \\
Soleimani
\end{tabular} & Iran/ 2019 & $\begin{array}{l}\text { To measure } \\
\text { the influence } \\
\text { of hard and } \\
\text { soft TQM } \\
\text { factors on } \\
\text { customer } \\
\text { behavior } \\
\text { based on the } \\
\text { role of } \\
\text { innovation } \\
\text { and } \\
\text { continuous } \\
\text { improvement. }\end{array}$ & $\begin{array}{l}\text { The population is } \\
\text { the workers in } \\
\text { the Hospital } \\
\text { consisting of } \\
\text { project managers, } \\
\text { quality assurance } \\
\text { managers as well } \\
\text { as quality control } \\
\text { managers. The } \\
\text { sample was } 374 \\
\text { respondents. }\end{array}$ & $\begin{array}{c}\text { Quantitativ } \\
\mathrm{e}\end{array}$ & $\begin{array}{l}\text { 1. Long- } \\
\text { term } \\
\text { commitm } \\
\text { ent } \\
2 . \\
\text { Continuo } \\
\text { us system } \\
\text { improve } \\
\text { ment }\end{array}$ & $\begin{array}{l}\text { 1. Hard } \\
\text { and soft } \\
\text { Total } \\
\text { Quality } \\
\text { Manageme } \\
\text { nt (TQM) } \\
\text { 2. Patient } \\
\text { behavior } \\
\text { 3. Patient } \\
\text { satisfaction }\end{array}$ & $\begin{array}{l}\text { TQM has a significant } \\
\text { influence on customer } \\
\text { behavior through quality } \\
\text { improvement and } \\
\text { continuous innovation } \\
\text { that impacts patient } \\
\text { satisfaction. }\end{array}$ \\
\hline 24 & $\begin{array}{l}\text { The impact } \\
\text { of TQM } \\
\text { practice on } \\
\text { patients' } \\
\text { satisfaction } \\
\text { through } \\
\text { operational } \\
\text { flexibility }\end{array}$ & \begin{tabular}{|c|} 
Main Naser \\
Alolayyan, \\
Khairul \\
Anuar Mohd \\
Ali, Fazli \\
Idris
\end{tabular} & $\begin{array}{c}\text { Yordania/ } \\
2020\end{array}$ & $\begin{array}{l}\text { To study the } \\
\text { impact of } \\
\text { TQM } \\
\text { implementati } \\
\text { on on patient } \\
\text { satisfaction } \\
\text { levels in } \\
\text { Jordanian } \\
\text { university } \\
\text { hospitals by } \\
\text { considering } \\
\text { operational } \\
\text { flexibility as } \\
\text { a mediation } \\
\text { variable. }\end{array}$ & $\begin{array}{l}\text { Population of } \\
\text { medical staff } \\
\text { working in } \\
\text { university } \\
\text { hospitals in } \\
\text { Jordan. The } \\
\text { population is } \\
\text { divided into } \\
\text { doctors, nurses, } \\
\text { and medical } \\
\text { technicians. }\end{array}$ & Qualitative & $\begin{array}{l}\text { 1. Focus } \\
\text { on the } \\
\text { patient } \\
2 \text {. Long- } \\
\text { term } \\
\text { commitm } \\
\text { ent } \\
3 \text {. } \\
\text { Teamwor } \\
\mathrm{k} \\
4 . \\
\text { Continuo } \\
\text { us system } \\
\text { improve } \\
\text { ment }\end{array}$ & $\begin{array}{l}\text { 1. Total } \\
\text { Quality } \\
\text { Manageme } \\
\text { nt (TQM) } \\
2 . \\
\text { Operationa } \\
\text { 1 flexibility } \\
\text { 3. Patient } \\
\text { satisfaction }\end{array}$ & $\begin{array}{l}\text { The study will clarify } \\
\text { the direct relationship } \\
\text { between TQM and } \\
\text { patient satisfaction, the } \\
\text { indirect relationship } \\
\text { between TQM and } \\
\text { patient satisfaction } \\
\text { through operational } \\
\text { flexibility and the } \\
\text { impact of operational } \\
\text { flexibility on patient } \\
\text { satisfaction. }\end{array}$ \\
\hline
\end{tabular}




\begin{tabular}{|c|c|c|c|c|c|c|c|c|c|}
\hline & & & & & & & $\begin{array}{l}5 . \\
\text { Educatio } \\
\mathrm{n} \text { and } \\
\text { training } \\
6 . \\
\text { Controlle } \\
\mathrm{d} \\
\text { freedom } \\
7 . \\
\text { Employe } \\
\mathrm{e} \\
\text { engagem } \\
\text { ent and } \\
\text { empower } \\
\text { ment } \\
\end{array}$ & & \\
\hline 25 & $\begin{array}{l}\text { The } \\
\text { influence of } \\
\text { soft skills, } \\
\text { total quality } \\
\text { management } \\
\text {, and } \\
\text { operational } \\
\text { performance } \\
\text { on the } \\
\text { quality of } \\
\text { service and } \\
\text { patient } \\
\text { satisfaction } \\
\text { at type B } \\
\text { hospitals in } \\
\text { Makassar } \\
\text { City }\end{array}$ & $\begin{array}{c}\text { Irianti, } \\
\text { Nuraini } \\
\text { Rasyid, } \\
\text { Widyawati }\end{array}$ & $\begin{array}{c}\text { Indonesia/ } \\
2021\end{array}$ & $\begin{array}{l}\text { To find out } \\
\text { the influence } \\
\text { of soft skills, } \\
\text { total quality } \\
\text { management, } \\
\text { and } \\
\text { operational } \\
\text { performance } \\
\text { on the quality } \\
\text { of service and } \\
\text { patient } \\
\text { satisfaction at } \\
\text { type B } \\
\text { hospitals in } \\
\text { Makassar } \\
\text { City. }\end{array}$ & $\begin{array}{l}\text { The population in } \\
\text { this study is a } \\
\text { type B hospital in } \\
\text { Makassar City. } \\
\text { The sample was } \\
398 \text { participants. }\end{array}$ & $\begin{array}{c}\text { Quantitativ } \\
\mathrm{e}\end{array}$ & $\begin{array}{l}1 . \\
\text { Obsessio } \\
\text { n with } \\
\text { quality } \\
2 . \\
\text { Teamwor } \\
\mathrm{k} \\
3 . \\
\text { Continuo } \\
\text { us system } \\
\text { improve } \\
\text { ment } \\
4 . \\
\text { Educatio } \\
\mathrm{n} \text { and } \\
\text { training }\end{array}$ & $\begin{array}{l}\text { 1. Total } \\
\text { Quality } \\
\text { Management } \\
\text { (TQM) } \\
\text { 2. Soft Skill } \\
\text { 3. } \\
\text { Operational } \\
\text { performance } \\
\text { 4. Quality of } \\
\text { service } \\
\text { 5. Patient } \\
\text { satisfaction }\end{array}$ & $\begin{array}{l}\text { TQM and partial } \\
\text { operational performance } \\
\text { have a positive and } \\
\text { significant impact on } \\
\text { service quality and } \\
\text { patient satisfaction. }\end{array}$ \\
\hline
\end{tabular}

The results of the overall literature that have been extracted can be seen in Table 1. All of the literature included in this study was published from 2016 to 2021, as many as 25 literatures.

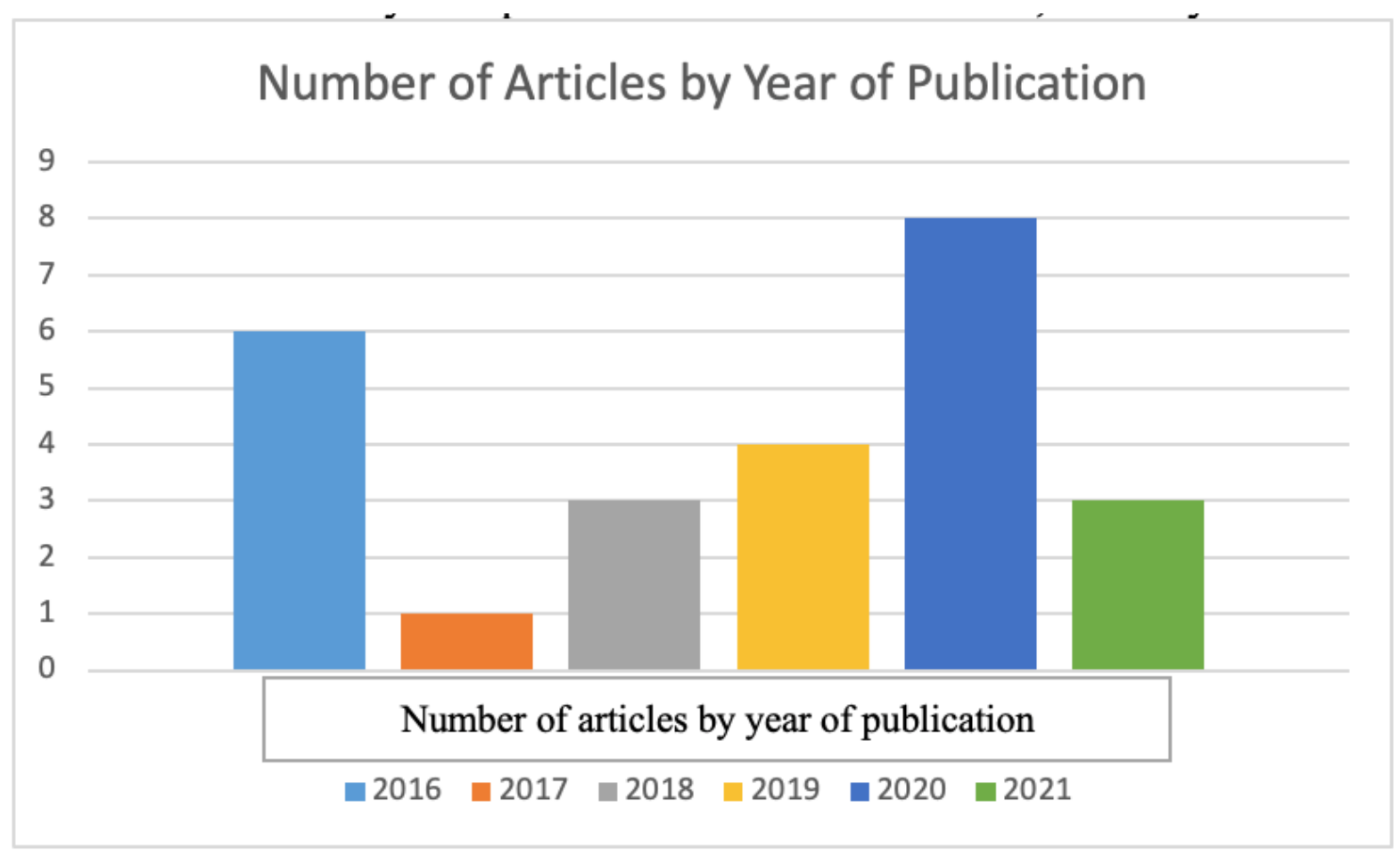

Figure 3. Number of Articles by Year of Publication

Based on the figure. 3 years of most publications in 2020 and 2016 while in 2017 only 1 article was published. 




Figure 4. Number of articles by country

In Figure 4. There are 2 countries with 3 articles in total, namely Vietnam and the UK, then Jordan, Iraq, Bangladesh, and Uganda with 2 articles, and other countries with only 1 article.

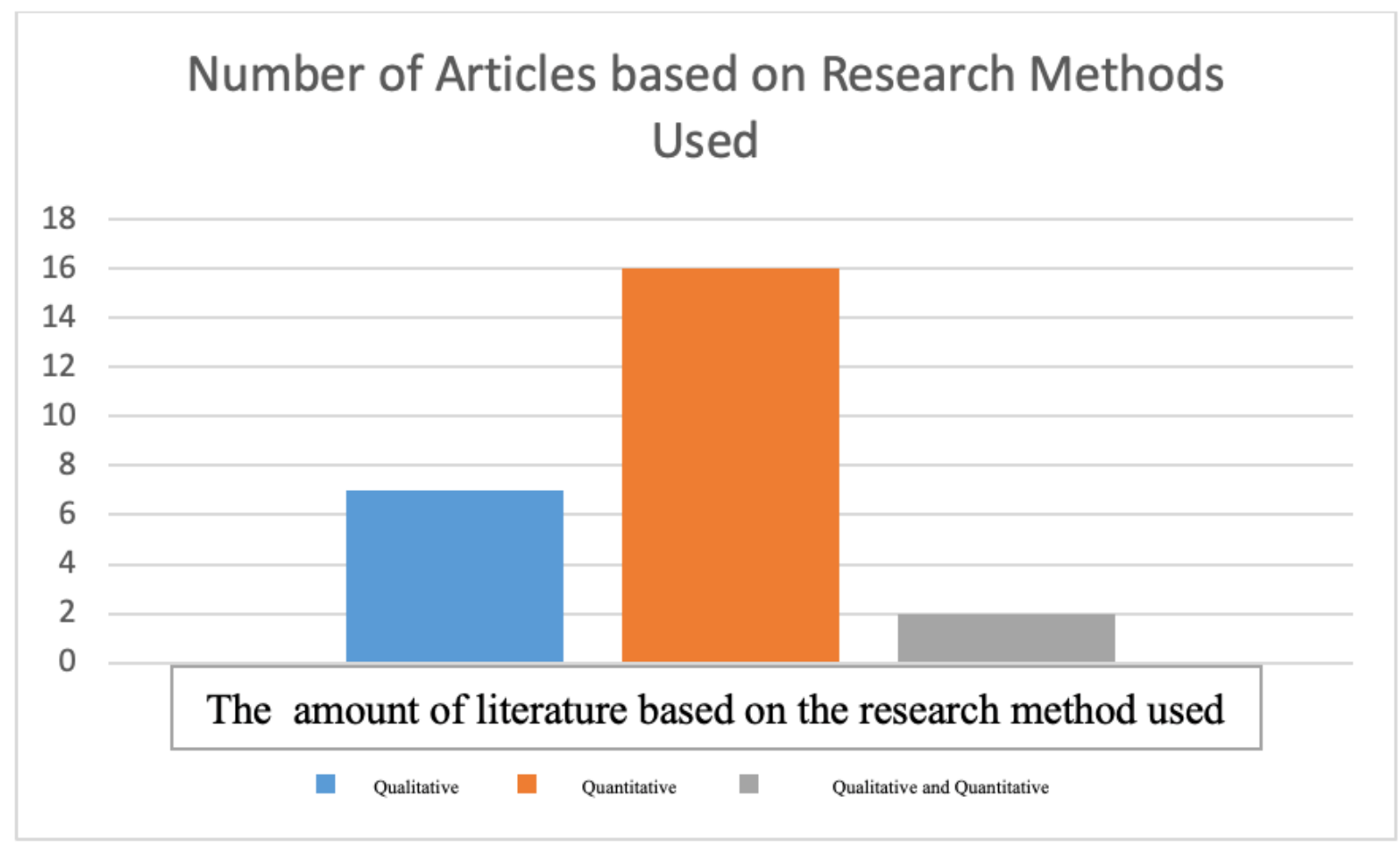

Figure 5. Number of Articles based on Research Methods used

Figure 5 depicts the number of articles based on the type of research, most of the research methods analyzed using quantitative methods are 16 articles, then qualitative methods are 7 articles, and qualitative and quantitative methods are 2 articles. Then, the authors mapped the search results of articles based on the type of innovation in Table 2. qualitative and quantitative 
Table 2. Mapping of TQM Elements that Affect Patient Satisfaction, Employee Satisfaction and Productivity in Hospitals

\begin{tabular}{|c|c|c|c|c|}
\hline $\begin{array}{l}\text { TQM Implementation } \\
\text { Elements }\end{array}$ & $\begin{array}{l}\text { Amount of } \\
\text { Literature }\end{array}$ & $\begin{array}{c}\text { Patient } \\
\text { Satisfaction } \\
\text { [source] }\end{array}$ & $\begin{array}{c}\text { Employee } \\
\text { Satisfaction } \\
\text { [source] }\end{array}$ & $\begin{array}{l}\text { Productivity } \\
\text { [source] }\end{array}$ \\
\hline Focus on the patient & 18 & $\begin{array}{c}4],[5],[6],[7], \\
{[9],[10],[12],} \\
{[13],[14],[15],} \\
{[16],[17],[18],} \\
{[19],[20],[21],} \\
{[22],[24]}\end{array}$ & [17] & $\begin{array}{c}{[2],[4],[5],} \\
{[14]}\end{array}$ \\
\hline Obsession with quality & 16 & $\begin{array}{c}4],[5],[6],[8], \\
{[9],[10],[12],} \\
{[13],[14],[16],} \\
{[17],[18],[19],} \\
{[20],[22],[25]}\end{array}$ & [17] & - \\
\hline Scientific approach & 1 & [5] & - & - \\
\hline Long-term commitment & 6 & $\begin{array}{l}{[4],[18],[20],} \\
{[21],[24],[25]}\end{array}$ & & [2] \\
\hline Teamwork & 8 & $\begin{array}{c}{[1],[4],[7],[8],} \\
{[20],[21],[24],} \\
{[25]}\end{array}$ & [1], [7] & [5] \\
\hline $\begin{array}{l}\text { Continuous system } \\
\text { improvement }\end{array}$ & 15 & $\begin{array}{c}{[3],[4],[6],[11],} \\
{[12],[13],[14],} \\
{[15],[16],[17],} \\
{[20],[22],[23],} \\
{[24],[25]}\end{array}$ & {$[6],[7],[17]$} & [3], [14], [24] \\
\hline Education and training & 7 & $\begin{array}{c}{[4],[5],[8],} \\
{[21],[22],[24],} \\
{[25]}\end{array}$ & [7] & [5], [14] \\
\hline Controlled freedom & 3 & {$[4],[24],[25]$} & - & - \\
\hline Unity of purpose & - & - & - & - \\
\hline $\begin{array}{c}\text { There is employee } \\
\text { engagement and } \\
\text { empowerment }\end{array}$ & 17 & $\begin{array}{c}2],[4],[5],[6], \\
{[7],[8],[9],[10],} \\
{[11],[14],[16],} \\
{[17],[20],[21],} \\
{[22],[24],[25]}\end{array}$ & [7], [17] & [2], [5] \\
\hline
\end{tabular}

Based on the analyzed articles, it was found that the elements of TQM that affect patient satisfaction in hospitals, and the elements that most influence patient satisfaction, as well as elements of TQM that affect employee satisfaction and productivity in hospitals, can be seen in Table 2.

According to Johnson et.al (1995) and Andaleeb (1995), patient satisfaction is defined as the customer's overall evaluation of his or her experience with hospital services. A legitimate way to improve patient care is customer satisfaction checks and monitoring. According to Vukmir (2006), the most important definition of overall patient satisfaction is when the patient's expectations for treatment and care are met or exceeded. In addition, Naidu (2008) defines 
patient satisfaction as an important indicator for the evaluation of different dimensions of health care (Naser Alolayyan, 2016)

The main objective in Total Quality Management is to maintain customer satisfaction at the highest level and to meet customer requirements. The concept of customer orientation is to provide services in accordance with customer demands. Before the product or service reaches the customer, all conditions must be reviewed and possible errors must be avoided. In today's increasingly competitive environment, the goal of all businesses is to work in a customeroriented manner and conduct various studies to attract customers to themselves. For this reason, awareness arises to get customers and aims to present products or services in the best way (Bilen \& Sitki, 2020)

The application of TQM strategies in the health sector is becoming increasingly popular as the benefits of TQM have been proven. Alexander et al (2008) suggested that implementing TQM has been shown to help better care according to patient needs, improve processes and reduce medical errors, thereby increasing patient satisfaction and productivity. Duggirala et al. (2008) confirmed that the application of TQM in hospitals helps identify key factors to improve patient satisfaction and helps to involve patients effectively in service planning (Alrabeah et al., 2020)

\section{The Effect of Patient Focus on Patient Satisfaction}

Customer focus is one of the basic requirements in Total Quality Management. The adoption of TQM requires healthcare organizations to design their products and pathways on the idea of customer satisfaction, or better. The main goal of TQM is customer satisfaction and focus on meeting client needs. Long-term plans to meet or exceed customer satisfaction expectations are essential. Juran (1993) asserts that there are two types of customers; external customers such as clients, government agencies, and the public sector and internal customers such as employees who determine quality and service providers (Psychogios \& Priporas, 2007) (Naser Alolayyan, 2016)

\section{The Effect of Obsession on Quality on Patient Satisfaction}

Zeyrekliyas (2009) stated that the purpose of TQM is to satisfy customers by determining customer needs and providing complete products or services according to these needs, and also making continuous efforts to improve quality. Based on this, the most important feature of TQM is to provide quality products and services by sharing with all employees. The success of TQM depends on its implementation by all employees and its determination from the top level employees to the lowest level (Bilen \& Sitki, 2020)

\section{Influence of Scientific Approaches to Patient Satisfaction}

According to Goetsch \& Davis (1994), a scientific approach is needed in the application of total quality management, especially for designing work and in the decision-making process and problem solving related to designing work.

In hospitals, a scientific approach is carried out to design work, decision-making processes and problem solving related to predetermined work using the data needed and used in establishing benchmarks to improve performance and carry out improvements. These improvements can be carried out on an ongoing basis so that the quality of service can be increased.

\section{The Effect of Long-Term Commitment on Patient Satisfaction}

According to Eroglu (2003), the success of Total Quality Management (TQM) is influenced by the commitment of top management. The more determined the top management, the leadership in this case will motivate employees positively. Leaders are people who have the ability to 
influence, move and guide their subordinates towards predetermined goals. Manager is a person who strives to achieve set goals, plan work, ensure execution and supervise. They have responsibilities based on rewards and punishments (Bilen \& Sitki, 2020)

\section{Effect of Teamwork on Patient Satisfaction}

A team is a collection of people. According to Chang (2001), these needs include: effective communication and active listening, resolving conflicts that inevitably arise when people work in groups, and maintaining motivation among all team members. For TQM to be fully functional, teamwork and participation is a strength, especially in medical services (Balasubramanian, 2016)

\section{The Effect of Continuous System Improvement on Patient Satisfaction}

The continuous improvement process is at the core of TQM, the introduction of continuous improvement in all areas of work in healthcare organizations in order to be able to permanently adapt to changes in the internal and external environment of health organizations. Continuous improvement is necessary for the success of TQM. It aims to achieve permanently and continuously in the production process of health organizations. Quality is endless, but it is a process that requires moving forward without pause (Muhaimeed et al., 2021)

\section{The Impact of Education and Training on Patient Satisfaction}

From the TQM idea, quality is the collective responsibility of all employees not managers (Klefsjö, Bergquist, \& Edgeman, (2006). Employees who have been included in the decisionmaking on the factors affecting their work environment and have been given adequate education and training. Support makes a huge contribution to the success of the TQM program (DATA 2) (Mwikali \& Bett, 2019). To make quality a way of life and a trend of thought, employees must attend an intensive training program aimed at the education of participants (Balasubramanian, 2016)

\section{Controlled freedom influences patient satisfaction}

According to Goetsch \& Davis (1994), employee involvement in decision making and problem solving in hospitals is an important thing in improving service quality. Based on research conducted by Abdulrahman (2020) by conducting interviews with management interviewed in all cases believed that there was strong employee involvement, citing departmental meetings as a forum for providing advice and engaging in discussions on issues. Employee involvement is one of the freedoms given by the hospital to its employees (Alrabeah et al., 2020)

\section{The effect of unity of purpose on patient satisfaction}

Based on the results of a journal review of 25 journals with a time span of the last 5 years, there were no journals that discussed the effect of unity of purpose on patient satisfaction in hospitals.

\section{The Effect of Employee Engagement and Empowerment on Patient Satisfaction}

To achieve patient satisfaction, it is necessary to continuously improve quality and involve employees in every process of the organization's activities. Without these two activities, the desire to provide satisfaction to patients will be difficult to achieve (Indra Abadi et al., 2018)

Goetsch and Davis (2013) identified that employees must be physically and emotionally involved in decision making and quality management. Employers and management should have open systems that encourage employee engagement, recognize employee values and involve employees in the service delivery process. Their participation is a fundamental tool in responsible HRM practices to improve quality. According to Naidu et al (2006), resistance can 
develop among employees when their engagement is undermined. This is justified because TQM requires commitment and a good attitude. Evans and Lindsay, (2008) highlight that when a manager has considered employees in decision making and provided encouragement to them to contribute to the success of the organization then their quality is guaranteed (Mwikali \& Bett, 2019)

\section{The elements of TQM that most affect patient satisfaction}

Based on the journal mapping that has been carried out, it was found that the most influential element of TQM is the Patient Focus aspect, where from the 25 journals found this aspect was discussed in more than 18 journals. With the average result that this element greatly affects the level of patient satisfaction in the hospital.

This is in line with the service system used in hospitals today. Namely Patient Centered Care (PCC), where PCC is a health care system that focuses on patients. This system places the patient as a subject in every care provided. Based on the results of research conducted by Wahyuni N P S \& Darmawan ES (2019) that the core concept of PCC is to treat patients as unique individuals, respect their values and beliefs, and respond flexibly to patient needs and preferences. The PCC approach is proven to improve patient status through better communication and to encourage interaction between patients and providers (Sri Wahyuni \& Darmawan, 2019)

\section{The Effect of TQM on Nurse Satisfaction}

Staff satisfaction is an important issue among healthcare professionals because it has been associated with the prediction of their turnover [25]. Moreover, there is a definite relationship between staff satisfaction and patient satisfaction. Knowledge of the factors that contribute to staff satisfaction can provide a useful framework for hospital management to create a better practice environment for all healthcare professionals. Therefore, it is important to evaluate staff satisfaction to identify problems and try to solve them before they impact on the quality of patient care. Reduced job stress, greater job autonomy, adequate staffing, camaraderie among members, fairness, communication with supervisors and coworkers and increased collaboration with medical professionals have all been shown to be associated with staff satisfaction. Leadership style is also directly related to staff satisfaction. Positive interprofessional relationships and collaboration between staff and leaders have been shown to increase staff satisfaction and team performance. However, teamwork has been considered an important contributor to providing an environment to encourage staff satisfaction (Hamed Khawka, 2016)

\section{Effect of TQM on Productivity}

According to Alexander et al (2006), implementing TQM has been shown to help better care according to patient needs, improve processes and reduce medical errors, thereby increasing patient satisfaction and productivity. Duggirala et al. (2008) confirmed that the application of TQM in hospitals helps identify key factors to improve patient satisfaction and helps to involve patients effectively in-service planning.

\section{Conclusion}

The elements of Total Quality Management (TQM) that affect patient satisfaction in hospitals are Patient Focus, Obsession with Quality, Scientific Approach, Long-term commitment, Teamwork, Continuous system improvement, Education and training, Controlled freedom, Involvement and Employee empowerment. The element of Total Quality Management (TQM) that most influences patient satisfaction in hospitals is the element of Focus on Patients. This 
is in line with the service system used in hospitals today, namely Patient Centered Care (PCC). The PCC approach has been shown to improve patient status through better communication and to encourage interaction between patients and providers. Elements of Total Quality Management (TQM) that affect employee satisfaction in hospitals are patient focus, obsession with quality, teamwork, continuous system improvement, education and training, controlled freedom, employee involvement and empowerment. Elements of Total Quality Management (TQM) that affect productivity in hospitals are patient focus, long-term commitment, teamwork, continuous system improvement, education and training, employee involvement and empowerment.

\section{References}

Adhiputra, M. W. (2019). Analisis Penerapan Tqm Terhadap Kinerja Manajerial Pada Rumah Sakit Umum Di Denpasar. Jurnal Ilmiah Akuntansi, 3(1), 49-60. https://doi.org/10.23887/jia.v3i1.16628

Alolayyan, M. N., Anuar, K., Ali, M., \& Idris, F. (2010). The Impact of TQM Practice on Patients'nsatisfaction through Operational Flexibility. 1-17.

Alrabeah, A. H., Ogden, S., Edgar, D., \& Fryer, K. (2020). Total Quality Management and Hospital Workforce National Cultural Diversity in Saudi Arabia: Help or Hindrance? International Journal of Research in Business Studies and Management, 7(5), 18-30.

Alzoubi, M. M., Hayati, K. S., Rosliza, A. M., Ahmad, A. A., \& Al-Hamdan, Z. M. (2018). Total Quality Management Intervention for Enhancing Nursing Commitment and Performance in Jordanian Hospital: Protocol of a Quasi-Experimental Study. International Journal of Advanced Scientific Research \& Development (IJASRD), 5(10), 01. https://doi.org/10.26836/ijasrd/2018/v5/i10/51001

Babu, F., \& Thomas, S. (2020). The relationship between total quality management practices and organisational image in the hospital industry: An empirical examination. International Journal of Productivity and Quality Management, 29(1), 1-23. https://doi.org/10.1504/IJPQM.2020.104517

Balasubramanian, M. (2016). Total Quality Management [TQM] in the Healthcare Industry Challenges, Barriers and Implementation Developing a Framework for TQM Implementation in a Healthcare Setup. Science Journal of Public Health, 4(4), 271. https://doi.org/10.11648/j.sjph.20160404.11

Bilen, A., \& Sitki, A. (2020). The Impact of Total Quality Management on Patient Satisfaction: A Field Study in the Health Centers of the Syrians in Gaziantep. Journal of Arts \& Social Sciences, 7(1), 11-20. https://doi.org/10.46662/jass-vol7-iss1-2020(11-20)

Essel, R. E. (2020). Assessing Total Quality Management (TQM) Effect on Hospital Performance in Ghana Using a Non-Probabilistic Approach: The Case of Greater Accra Regional Hospital (GARH). Metamorphosis: A Journal of Management Research, 19(1), 29-41. https://doi.org/10.1177/0972622520949091

Hamed Khawka, Z. M. (2016). Detecting Total Quality Management Status and Teamwork Orientation in Al-Yarmouk Teaching Hospital. American Journal of Industrial and Business Management, 06(03), 232-248. https://doi.org/10.4236/ajibm.2016.63021

Hayat, N. N. (2020). Hubungan Karakteristik Staf Dan Tim Kerja Dengan Kepuasan Kerja Perawat Di Rumah Sakit Tipe B Tahun 2015. Human Care Journal, 5(1), 365. https://doi.org/10.32883/hcj.v5i1.686 
Indra Abadi, . M. H., . B. S., \& . A. M. (2018). A structural model of total quality management, kaizen, operational performance on service quality and patient satisfaction. Archives of Business Research, 6(11), 48-60. https://doi.org/10.14738/abr.611.5590

Juran, J. M., Godfrey, a B., Hoogstoel, R. E., \& Schilling, E. G. (1999). Juran ' S Quality Handbook. In Training for Quality (Vol. 1, Issue 3). http://knovel.com/web/portal/browse/display?_EXT_KNOVEL_DISPLAY_bookid= $623 \&$ VerticalID $=0$

Juran, J., \& Godfrey, A. (1998). JURAN'S QUALITY HANDBOOK, 5th EDITION. In JURAN'S QUALITY HANDBOOK, 5th EDITION.

Kafidzin, R. (2014). Analisis implementasi. 1-20.

Kamra, V., Singh, H., \& Kumar De, K. (2016). Factors affecting patient satisfaction: an exploratory study for quality management in the health-care sector. Total Quality Management and Business Excellence, 27(9-10), 1013-1027. https://doi.org/10.1080/14783363.2015.1057488

Khawka, Z. M. H. (2016). Total Quality Management and Its Applications. American Journal of Industrial and Business Management, 6(3), 233-248. http://iarjset.com/upload/2017/may-17/IARJSET 30.pdf\%0Ahttp://www.scirp.org/journal/PaperDownload.aspx?DOI=10.4236/iim.20 13.54013\%0Ahttp://www.scirp.org/journal/doi.aspx?DOI=10.4236/jss.2016.411017

Masinambow, R., \& Karuntu, M. (2019). Analisis Penerapan Total Quality Management Di Rumah Sakit Siloam Gmim Sonder Analysis of the Application Total Quality Management in Siloam Gmim Sonder. Analisis Penerapan Total Quality Management Di Rumah Sakit Siloam Gmim Sonder Analysis of the Application Total Quality Management in Siloam Gmim Sonder, 7(1), 621-630.

Mawuko, D. W. (2017). The effect of total quality management practices on customer satisfaction in the upper west regional hospital.

Muhaimeed, K. A., Hawas, T. A., \& Mohammed, A. A. (2021). The availability of Total Quality Management requirements and contribution to the achievement of the quality of service health analytical study at Salah al-Din General Hospital.

Mwikali, E., \& Bett, S. (2019). Total quality management practices and service delivery at the Nairobi Hospital, Kenya. ... Journal of Human Resource and Business ..., 3(7), 572589. https://iajournals.org/articles/iajhrba_v3_i7_572_589.pdf

Naser Alolayyan, A. M. H. A. (2016). the Impact of Total Quality Management ( Tqm ) Practice on Patients ' Satisfaction Through Operational Flexibility. International Educational Scientific Research Journal, 2(7), 16-21. https://doi.org/10.21276/2455295

Nguyen, T. L. H., \& Nagase, K. (2020). Total Quality Management: A Mediating Factor in the Relationship Between Customer Expectations and Satisfaction. International Journal of Management and ..., 13(1), 1-13. https://papers.ssrn.com/sol3/papers.cfm?abstract_id=3736225

Pardede, J. . (2017). Analisis Komparatif Implementasi Program Total Quality Management (TQM) Dan Kepuasan Pasien Rawat Inap Dan Rawat Jalan (Studi Kasus paa Rumah Sakit Bina Kasih Dadn Rumah Sakit Lancang Kuning). JOM Fekom, 4(1), 10751089. 
Rahman, M., \& Raju, V. (2020). Patients'Perceptions on Factors Determining of Total Quality Management (Tqm) Implementation Into Bangladeshi Public Hospitals .... PalArch's Journal of Archaeology of Egypt ..., 17(7), 6258-6272. https://archives.palarch.nl/index.php/jae/article/view/2963

Sri Wahyuni, N. P., \& Darmawan, E. S. (2019). Patient Centered Care Model to Improve the Quality and Safety of Patient Care in Hospital: A Systematic Review. 291-291. https://doi.org/10.26911/the6thicph.04.53

Sriwidadi, T. (2001). Manajemen mutu terpadu. 2, 107-115.

Take, N., Byakika, S., Tasei, H., \& Yoshikawa, T. (2016). The effect of 5S-continuous quality improvement-total quality management approach on staff motivation, patients' waiting time and patient satisfaction with services at hospitals in Uganda. Journal of Public Health in Africa, 6(1), 29-34. https://doi.org/10.4081/jphia.2015.486

Wang, K. Y., Chou, C. C., \& Lai, J. C. Y. (2019). A structural model of total quality management, work values, job satisfaction and patient-safety-culture attitude among nurses. Journal of Nursing Management, 27(2), 225-232. https://doi.org/10.1111/jonm.12669 